\title{
Using Connectivity to Explain Autism
}

\author{
J.H. Adamek, BS ${ }^{\mathrm{a}}$; Y. Luo, BS ${ }^{\mathrm{a}, \mathrm{b}} ;$ J.B. Ewen, MD ${ }^{\mathrm{a}, \mathrm{c}}$
}

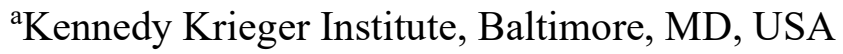

${ }^{b}$ Beihang University, Beijing, China

${ }^{c} J o h n s$ Hopkins University, Baltimore, MD, USA

The authors report no actual or apparent conflict of interest.

A version of this chapter is intended to be published in the upcoming Springer Handbook of Neuroengineering, N.V. Thakor (ed.). 


\section{SUMMARY}

The chapters in this Handbook reveal the breadth of brilliant imaging and analysis techniques designed to fulfill the mandate of cognitive neuroscience: to understand how anatomical structures and physiological processes in the brain cause typical and atypical behavior. Yet merely producing data from the latest imaging method is insufficient to truly achieve this goal. We also need a mental toolbox that contains methods of inference that allow us to derive true scientific explanation from these data. Causal inference is not easy in the human brain, where we are limited primarily to observational data and our methods of experimental perturbation in the service of causal explanation are limited. As a case study, we reverse engineer one of the most influential accounts of a neuropsychiatric disorder that is derived from observational imaging data: the connectivity theories of autism spectrum disorder (ASD). We take readers through an approach of first considering all possible causal paths that are allowed by preliminary imagingbehavioral correlations. By progressively sharpening the specificity of the measures and brain/behavioral constructs, we iteratively chip away at this space of allowable causal paths, like the sculptor chipping away the excess marble to reveal the statue. To assist in this process, we consider how current imaging methods that are lumped together under the rubric of "connectivity" may actually offer a differentiated set of connectivity constructs that can more specifically relate notions of information transmission in the mind to the physiology of the brain. 


\subsection{Introduction}

\subsubsection{A Challenging Goal: Causal Explanation}

This is a chapter about cognitive neuroscience- how we establish causal links that explain human behavior as a function of brain activity. Brilliant advances over the last few decades in both imaging technology and analysis methodology have extended our ability to record brain physiology in health and disease. Many interesting physiological phenomena have been observed, described, replicated and categorized. In some cases, replicable correlations have been demonstrated between observable measures of brain function and measures of behavior.

But correlation does not imply causation: because some imaging measure $I$ correlates with some behavioral measure $B$ does not automatically mean that the brain generator of $I$ causes behavior $B$. There are many causal paths that can generate the statistical relationship between $I$ and $B$. Nevertheless, some relationship causes this correlation, whether said relationship is scientifically interesting (true brain-behavior relationship) or trivial (artifactual confound). It is the goal of this chapter to consider our mental toolbox for constructing and refining the space of all possible relationships between these two domains, in order to achieve true causal explanations.

To clarify, the goal of reductive science is to explain how phenomena at a "smaller" level of analysis, such as brain physiology, cause phenomena at a "larger" level of analysis, such as behavior. That levels of analysis exist [1-4] and have such a causal relationship is the reductive assumption $^{1}$ (Fig. 1) [6 (Ch. 11), 7] that commonly underlies investigations of the mind-brain relationship.

\footnotetext{
${ }^{1}$ There is no consensus that this assumption is valid under all circumstances [5].
} 

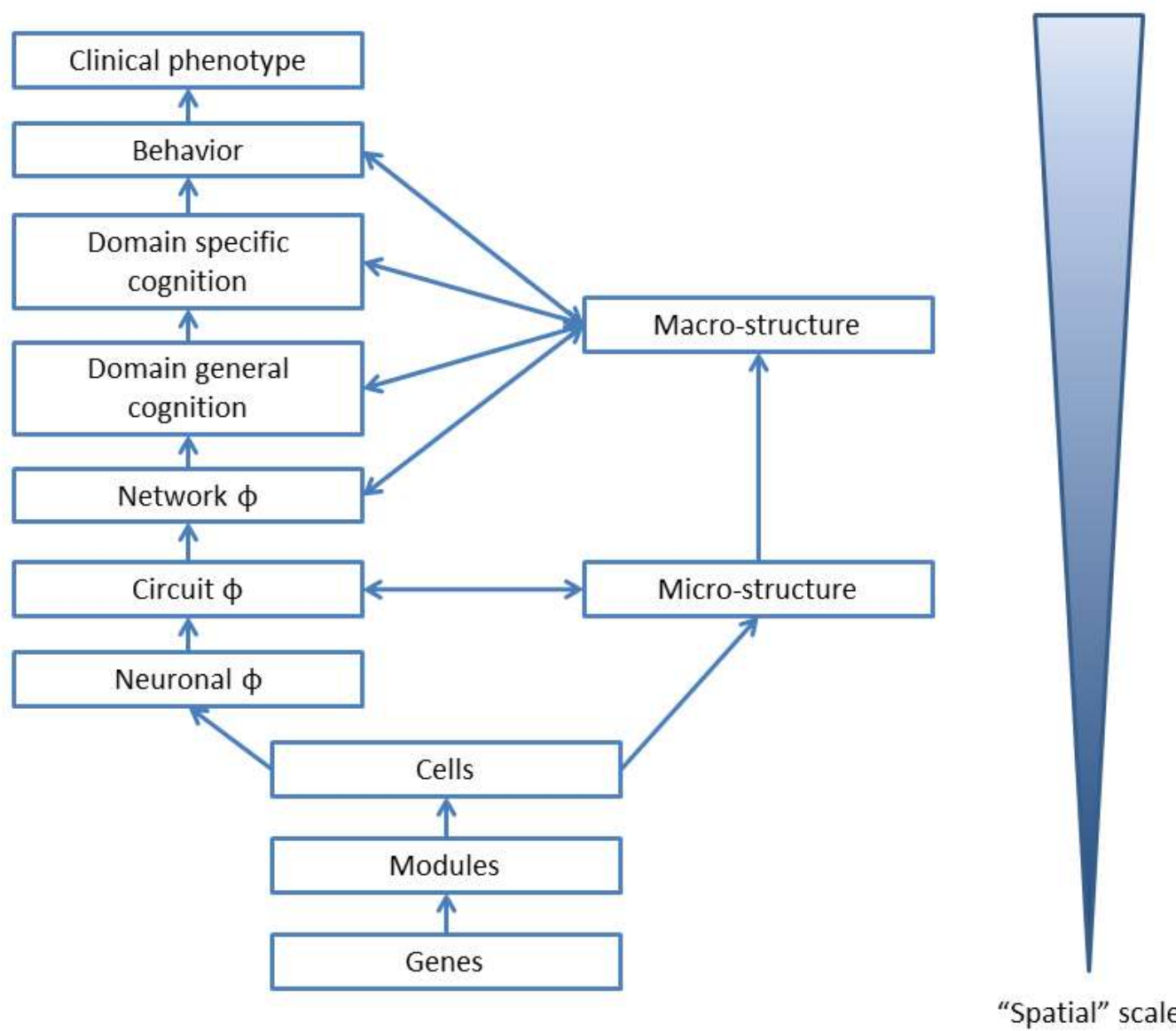

Figure. 1. Levels of Analysis in Neuroscience. There are no agreed upon, "canonical" levels of analysis in neuroscience, and the levels explicated within the context of an individual project are often generated ad hoc by the investigators. Nevertheless, the concept of levels of analysis permeates reductive science generally, including neuroscience. The specific set of levels of analysis presented here is relevant to connectivity-related research in ASD. We note also that environmental influences could act at many of these levels. By "behavioral," we follow the usage of Morton [3], who implicitly defines the behavioral level as that of observable behavior, including performance on psychometric tests or experimental tasks. The cognitive level is defined as the latent level of theoretical and computational processes contributing to behavior. We recognize that other usages of the words "cognitive" and "behavioral" exist in other contexts. " $\Phi$ " represents physiology.

The example we use is to illustrate causal inference is based around several observations dating to the early days of structural and functional connectivity (FC) imaging in which individuals with autism spectrum disorder (ASD) showed group differences in so-called connectivity measures and even that imaging connectivity measures correlate with symptom severity. But imaging measurements, behavioral observations and the correlations between them do not constitute true 
explanations of how behavioral features of the condition are caused by alterations of brain connectivity.

The traditional way to establish causality is to systematically manipulate the independent variable, randomly across subjects. However, in this context, we would have to cleanly and directly perturb cerebral connectivity over the course of development and assess downstream, ASD-related behavioral effects. We certainly do not have any methodological way to do so, and ethics would prohibit this line of investigation even if we did. Given that we mostly have only observational tools at our disposal, how can we attempt to establish a causal relationship?

Here, we need theory and hypotheses. And what we will see in the case study below is that observations documenting altered connectivity imaging measures in ASD and their relationship to behavior spawned a set of hypotheses proposing causal brain-behavior links. The approach to assessing these causal brain-behavior hypotheses using only observational data, then, is to propose and falsify as many alternative causal models as possible, iteratively updating our knowledge and approach as results accumulate. This is akin to revealing the true "statue of marble" by chipping away at the "false" material surrounding it [8]. And the best approach to generate sufficiently nuanced candidate causal graphs (hypotheses) is to increase the specificity of both brain and behavioral observations.

\subsubsection{Tool 1: Increasing the Specificity of Observations}

In order to arrive at the clearest conclusion, we sharpen our chisel by generating candidate causal models with the highest possible degree of specificity, comprised of elements with an optimal degree of specificity. The rough slab from which we carve our statue is demarcated by initial descriptive observations. Of course it is logically necessary for there to be group differences in connectivity if connectivity alterations truly cause some or all aspects of the ASD behavioral phenotype. For the case study of ASD, we start on solid ground: several recent reviews have indeed suggested the existence of diagnostic differences in imaging measures of connectivity [9-12]. Yet the data papers examined in these reviews reflect a jumble of different imaging technologies, analysis methods, regions of interest (ROI), task conditions, inclusion/exclusion criteria, subject-level covariates, performance measures, and even whether 
connectivity metrics were relatively increased or decreased in the ASD group. It is self-evident that we must care scientifically about whether ASD-related behavior is a result of increased connectivity vs. decreased connectivity! Some investigators use meta-analysis to begin to resolve our picture of the ASD-connectivity relationship. Yet meta-analyses fill a need that assumes that the gap in our understanding is lack of statistical power and that the choices of imaging modality, analysis method, etc., are largely irrelevant to the conclusion. This is not what we are after. Instead, to sharpen our understanding, we need a more nuanced understanding of the relationship between experimental parameters and observed variables: which participant characteristics or brain areas or task conditions tend toward null imagining results and which do not. The reviews cited above have begun to parse these parameters. Work that examines drastic shifts of FC as a function of age is highlighted in section 1.1.5; this is critical in understand the role of development in the connectivity-ASD story. In section 1.2, we will see how specific observations have motivated specific causal accounts.

\subsubsection{Tool 2: Theoretical Constructs and Causal Models}

To make causal inferences, it is not adequate only to increase the specificity of those variables we can measure directly: participant characteristics, imaging metrics, behavioral measures. For one thing, if we simply proclaim that, because connectivity correlates with a certain behavior, it therefore causes that behavior, there are at least four alternative causal relationships we need to examine. The first is that the measured biology truly causes the specific behavior. The second is that a methodological artifact generates a confounding and false association; see section 1.1.5 and Figure 2 for an example. The third is that connectivity changes could be compensatory to or downstream from the truly causal brain basis of the behavior. See section 1.2.4 and Figure 6 as an example of altered long-range connectivity as a downstream effect of the pathogenic process. The fourth is that the measured connectivity difference is epiphenomenal to the target behavioral differences: i.e., both are downstream from a common factor, but connectivity is not within the causal chain that produces that behavior. See section 1.2.1 and Figure 4 for an example.

To discern among these possibilities, we need causal graphs with adequate internal structure. And because there is a limit to the number of constructs that we can measure directly with 
current tools, we need to generate theoretical (inferred) terms - terms that cannot be observed/measured directly [13]. The principal theoretical term that we need to consider deeply is that of connectivity itself. When neuroscientists speak about "connectivity," what we have in mind is a range of theoretical notions about how behaviorally-relevant information flows through the computational mind, from one physical brain region to another. Dennett and others refer to such functional information as "semantic" information $[14]^{2}$. To the neuroscientist, "connectivity" does not simply mean a set of bivariate or network analysis tools computed on physiological imaging data and so named simply because they have an assumed relationship to brain function. Yet in practice, the use of the term seems to dangerously conflate the two - as if some new so-called connectivity technique will finally settle all inconsistencies in the ASDconnectivity literature. Rather, what will actually settle inconsistencies in the literature and advance understanding is a more nuanced testing of the theoretical constructs of connectivity (Table 1) - ones which predict that we should see changes in connectivity measures in only frontal-parietal connections (see section 1.2.5) vs. all connections (see section 1.2.2), or whether we should see differences during "higher-order" cognitive tasks and not "lower-order" cognitive tasks (see section 1.2.3) vs. even during rest (1.2.1). Nuanced predictions further derive from nuanced theoretical sub-constructs of connectivity; this is seen in some models which we review critically (e.g., sections 1.2.5 and 1.2.6) and is the major focus of section 1.3, in which we measure sub-constructs of semantic connectivity using several measures not traditionally thought of as "connectivity measures."

As we shall see in section 1.2, there are many other theoretical terms that are used to bind together observable measurements into scientific hypotheses and causal graphs. Theoretical constructs exist at both a cognitive level, inferred from behavioral data, and a "brain theory" level, inferred from imaging data. When we begin to invoke these theoretical terms, we (consciously or not) take on the axiomatic assumptions and external evidence of construct validity from the disciplines which gave birth to those terms. The implications of employing theory are non-trivial and are discussed in sections 1.1.4 and 1.1.5. In addition, these sections

\footnotetext{
2 "Semantic" in this instance refers to functionally relevant information and not to the way the term is used in linguistics or philosophy of science.
} 
also cover the behavioral and imaging phenomenology in ASD, around which we base our theories.

\subsubsection{Behavioral Phenomenology, Cognitive Theories and ASD}

ASD as we understand it today was first described in 1943 by Kanner [15], and is currently recognized in $1.7 \%$ of children [16]. It is diagnosed in the presence of two core behavioral features: social communication/interaction deficits and repetitive actions/restricted interests [17]. Individuals with ASD also have higher-than-expected rates of additional (non-diagnostic) cognitive atypicalities, including alterations of attention, executive function [18], language, general intelligence, motor skills [19], memory and perception (sensory hyper- and/or hyposensitivity).

Behavior is what our neurobiological causal models are trying to explain: the explanandum. The most satisfactory models would explain the diagnostic features of the disorder. However, this has been a challenge because there is no single account of ASD-related social deficits - no single behavioral metric or psychological construct or brain network to latch onto. Even rating scales and observational tools used to capture ASD for the clinical and research purposes anecdotally have challenges with inter-rater and inter-instrument reliability. Moreover, the condition is studied within a range of psychological disciplines (e.g., developmental psychology, neuropsychology, linguistics, ecological psychology), each with its own methodologies, assumptions and theoretical constructs [20]. Some fields have rigorous procedures for validating theoretical terms $[1(\$ 3.3), 21,22]$, whereas others seem to add them ad hoc. In the models

presented in section 1.2, we generally follow the authors' use of terminology, but work that will advance the field will need to bring constructs into communion in order to test models in competition.

One important example to illustrate how assumptions materially impact the framing of brainbehavior models: Many psychological disciplines try to reduce social (dys)function to more fundamental psychological domain, such as executive function [23]. This reduction is not only explanatory in its own right and possibly helpful for designing therapies, but also helps explain the correlation between social deficits and alterations of other, non-social abilities, e.g., 
perception and motor function. In imaging studies, we can also take advantage of the fact that brain networks involving executive function are better characterized than social networks. Contrasting frameworks take social deficits as modular and irreducible — an innate ability [24, 25]. Under this assumption, examining the executive function networks would therefore be relevant only to understanding executive dysfunction within ASD and not understanding the core social features themselves. The reader will note that most of the causal accounts in section 1.2 assume some sort of reducibility of social function. As we choose the explanandum, we therefore do so because the relevant behavioral phenomenon is either felt to be clinically relevant in its own right or causally relevant to the core features of the disorder.

We further note that ASD is characterized by high levels of heterogeneity: some individuals with ASD have language deficits and others do not. Many have executive function deficits, but some do not. Some have sensory hypersensitivity and others have sensory hyposensitivity [26]. It is therefore likely that there are multiple causal pathways to the ASD diagnostic phenotype within latent subgroups. A practical path forward, then, is to reduce the explanatory scope of the candidate model: not to use connectivity try to explain ASD as a whole or even core social deficits, but to explain only a single non-diagnostic behavioral feature of ASD (“endophenotype”) [27]. This approach allows for greater internal specific of the model, and also sidestepping the problem of heterogeneity to a degree.

\subsubsection{Imaging Phenomenology, Theories of Brain Function and ASD}

Just as our explanatory models depend on the assumptions and constructs of our "parent" psychological discipline, so do they depend on our framework for thinking about brain function. Much of the early work in cognitive brain imaging was based on a localizationist, feed-forward neuropsychological model [21, 28, 29, 30 (Ch. 1-4), 31]. While neuropsychological (lesion) data and physiological data are not fully commensurable - e.g., bilateral activation of networks, via fMRI, when only one side is known to be necessary, as established by lesion studies [32] - the shared localizationist nature of the two techniques (pathology and imaging) have made them a good fit [1]. 
However, resting-state networks [33] and connectomics/graph theoretical networks [34] have offered alternative lenses into the macro-structure of brain physiology. The differences among these approaches is in part a function of what brain activity is considered most relevant but may also represent axiomatic stances about the nature of brain function. Indeed, the history of neuroscience has swung back and forth been between localizationism and holism [1]. Current trends encompass the whole range from lay-press caricatures of localizationism ("Scientists discover brain region responsible for...”) to dynamic systems (“Every neuron in the brain participates in every single computation."3). Regional, bivariate measures are typically used to evaluate localizationist claims, whereas graph theoretical measures fit well with holist views of brain function. Yet other theories focus on EEG oscillations of different frequencies as the constituent elements [35]. In summary, there is no single, agreed upon and substantiated connectivity-based model of brain function upon which to base sub-models that specifically explain the features of ASD. Moreover, investigators working in one discipline or under one set of assumptions may not even realize that conclusions from other groups may be based on a different set of assumptions. As we will see in the examples in 1.2, the choice of analysis method may indicate certain assumptions about brain function.

Whenever science develops a new lens, reams of data and decades of effort are needed to sort observations into meaningful groups. These are the observations from which theories are born [36]. As we will see in section 1.2, ASD theories involving connectivity evolved in parallel with novel "connectivity" imaging methods. Given our new imaging tools, it makes sense that much of the current work is descriptive. We wish to highlight two important descriptive studies of ASD which have changed frames of reference.

The work of Uddin and colleagues [37] tackled the question of why some resting-state studies showed decreases in connectivity metrics in ASD, whereas others showed increases. They concluded that age and pubertal stage could be responsible for these inconsistencies. Such findings, once validated, could lead to developmental hypotheses about altered and even pathogenic network activity in ASD.

\footnotetext{
${ }^{3}$ Recalled to have been spoken by Peter Robinson at the 2015 meeting of the Organization for Human Brain Mapping
} 
In another example, which cast doubt over a large number of ASD-connectivity results, Power and colleagues [38] have demonstrated that connectivity results can be confounded by movement artifact. Because children with ASD may move in the MRI scanner more than controls, their long-range connectivity read-outs may be artificially depressed, leading to false-positive results of decreased connectivity (Fig. 2).
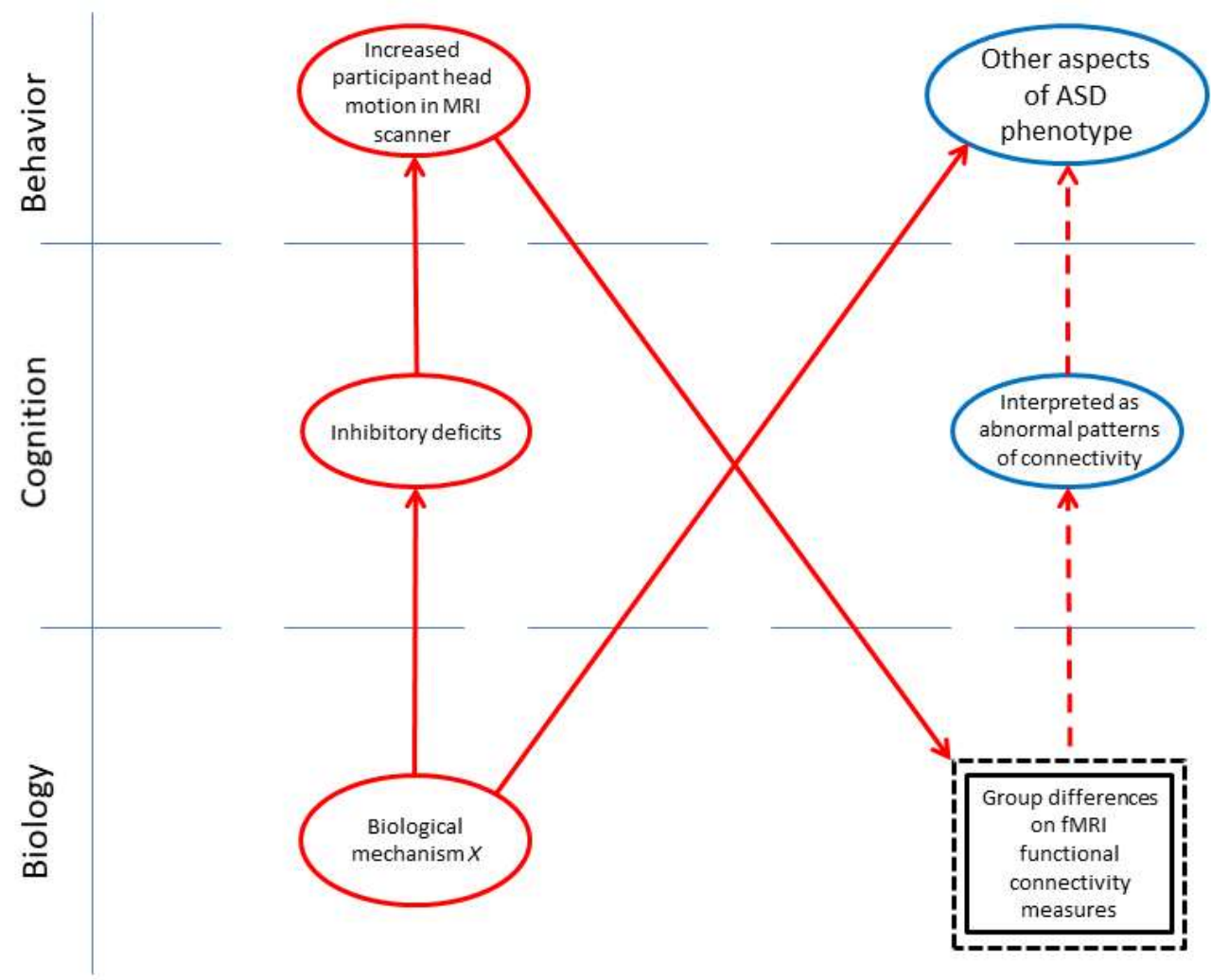

Figure 2. Correlation Does Not Entail Causation, Following the Results of Power et al. (2012). Behavioral aspects of the ASD phenotype are falsely attributed (dashed lines) to group differences in connectivity. What is actually happening is that ASD-associated increases in head motion are caused by an unstated mechanism ("mechanism X") and result in spuriously decreased connectivity measures. N.B.: In the causal model figures in this chapter, we generally following the approach of Morton [3], including biological factors, cognitive factors and behavioral factors. "Behavior" refers to observable psychological terms, while "Cognition" refers to theoretical psychological terms. This rubric does not distinguish between observable and theoretical biological terms, but we have made this distinction by putting observable elements in boxes surrounded by dashed lines. Some computational constructs seem to straddle biological levels and cognitive levels and are therefore placed on the boundary. 
To summarize, in the absence of the ability to perturb connectivity directly and measure the behavioral consequence, the best approach to approximate causality is to construct and falsify as many candidate causal paths as allowed by observed correlations between brain measures and behavior. To do so successfully, we rely on increasing the specificity of both our brain and behavioral measures. We first specify a behavioral explanandum: the behavioral feature that the physiology is believed to cause. The best explananda have relevance to the core of the disorder. We next use behavioral, brain and correlational observations to draw a boundary around the possible causal models. As the specificity of these observed variables increases, the volume of possible models shrinks. Adequate statistical power and other good scientific practices are critical at this stage [39]. Next, the definition and use of theoretical constructs (both brain and cognitive) is necessary to associate observable terms in a meaningful way within causal models. Doing so requires an examination of the inevitable assumptions behind those constructs. And doing so well allows us to integrate multimodal data and liberates us from pseudo-constructs caused by carelessly used language [40]. From this point, causal models can be tested in competition - including "scientifically interesting" models (as presented in section 1.2) or "scientifically trivial," confounded models (e.g., Figs. 2 and 4).

Readers may proceed in one of multiple ways. For those interested specifically in the ASDconnectivity story, read section 1.2 completely. For those who merely want the gist, the relevant points for understanding this approach to explanation are summarized in sub-section 1.2.7; refer to the rest of the section as needed. For those who are interested in our proposal for subconstructs of connectivity and how to measure them should read section 1.3; others may skip directly to section 1.4 .

\subsection{Causal Models of Connectivity, Cognition and Behavior in ASD}

\subsubsection{Horwitz et al. (1988)}

The first proposal that the ASD behavioral phenotype results from altered brain connectivity dates to 1964 and the dawn of the cognitive (rather than psychoanalytical and behaviorist) study of the condition [41]. This account is not covered in greater detail here because it did not clearly impact subsequent ASD-connectivity researchers. They were either not aware of this hypothesis 
or possibly considered the proposed biology — that cortical connectivity is mediated by the reticular formation - a brainstem system - to be hopelessly incorrect.

The first empiric investigation of connectivity in ASD was published by Horwitz and colleagues in 1988 [42]. This work, seemingly exploratory in its conception, occurred in the opening years of $\mathrm{FC}^{4}$ research via studying statistical associations between different brain regions' physiological read-outs $[43,44]$. This approach is the backbone of the current-day scientific enterprise of functional-connectivity MRI (fcMRI) as well as most EEG connectivity studies.

Up to that point, cortical connectivity had been studied anatomically, using the microscope and molecular tracers, and functionally, as it related to the production of behavior, through lesion studies [28-31]. At the time, an absence of a clear biological explanation for the ASD welcomed the use of a new technique with the hope of filling this gap in knowledge. Horwitz and colleagues used Positron Emission Tomography (PET) imaging to assess regional glucose metabolism, a measure of functional activation, in 59 different ROIs across the frontal lobes, parietal lobes, and several subcortical areas, as well as the correlations in glucose metabolism between pairs of ROIs. Comparing their sample of fourteen adult males with ASD and fourteen neurotypical adults, they found reduced correlations of glucose metabolism in 604 of the 861 measured region-of-interest (ROI) pairs in the ASD group, which they labeled, without further interpretation, as "reduced functional associations."

Based on the results, they speculated how decreased inter-regional correlation of brain activity could relate to "lower-level" neuronal processes and "higher-level" cognitive/behavioral processes, focusing specifically on an attentional hypothesis by Damasio and Maurer [45]. Previous research had shown reduced levels of cell death in the brain of individuals with ASD, which leads to an over-abundance of neuronal connections. The presence of too many neuronal pathways between distant brain regions could create abnormal functional organization, leading to inefficient information transfer and, subsequently, decreased glucose metabolism correlations

\footnotetext{
${ }^{4}$ We may have preferred the term "physiological connectivity" to label what is generally called FC: namely, the magnitude of statistical similarity between two or more physiological time-series. To us, the term "functional" refers to the production of action or goal-directed behavior-very much in the spirit of the causal brain-behavior relationship! But the term FC is often used to discuss physiological results independently of any discussion of behavior.
} 
between regions. Abnormal functional organization could, in turn, alter the mutually inhibitory balance between frontal and parietal regions responsible for mediating the approach-avoidance relationship with the surrounding environment. In their model, the frontal lobe ("responsible for" inward focus/environment avoidance) dominates the parietal lobe ("responsible for" outward focus/environmental approach), causing individuals with ASD to show increased inward-focused attention while largely ignoring their surroundings (Fig. 3).

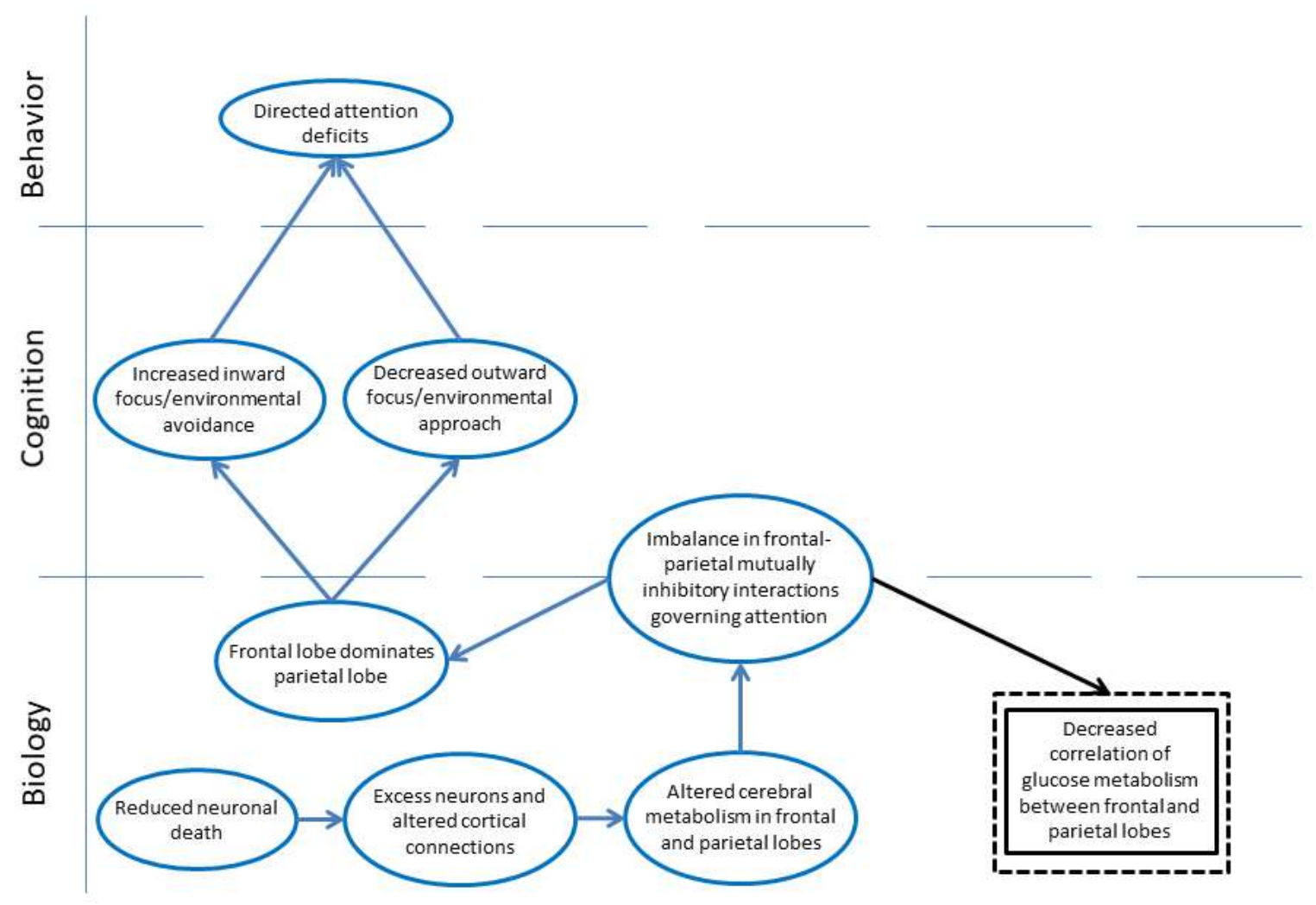

Figure 3: Causal model proposed by Horwitz and colleagues to explain specific attentional deficits present in ASD using altered FC as measured by inter-regional PET correlations. Note that these causal models have been drawn up by the authors of the current chapter. We only hope that they do justice to the intent of the authors of the original papers.

Their approach was exploratory, their results descriptive, and their causal model a posteriori. As a result of these limitations, the results were not able to exclude alternative, plausible causal accounts and admit some confounds: we note that the two groups differed based on IQ; current accounts of the neurobiology of intelligence suggest that general intellectual function may 
depend on frontal-parietal connectivity [46]. Might differences in IQ rather than in ASD phenotype have led to the group differences in PET results (Fig. 4)?

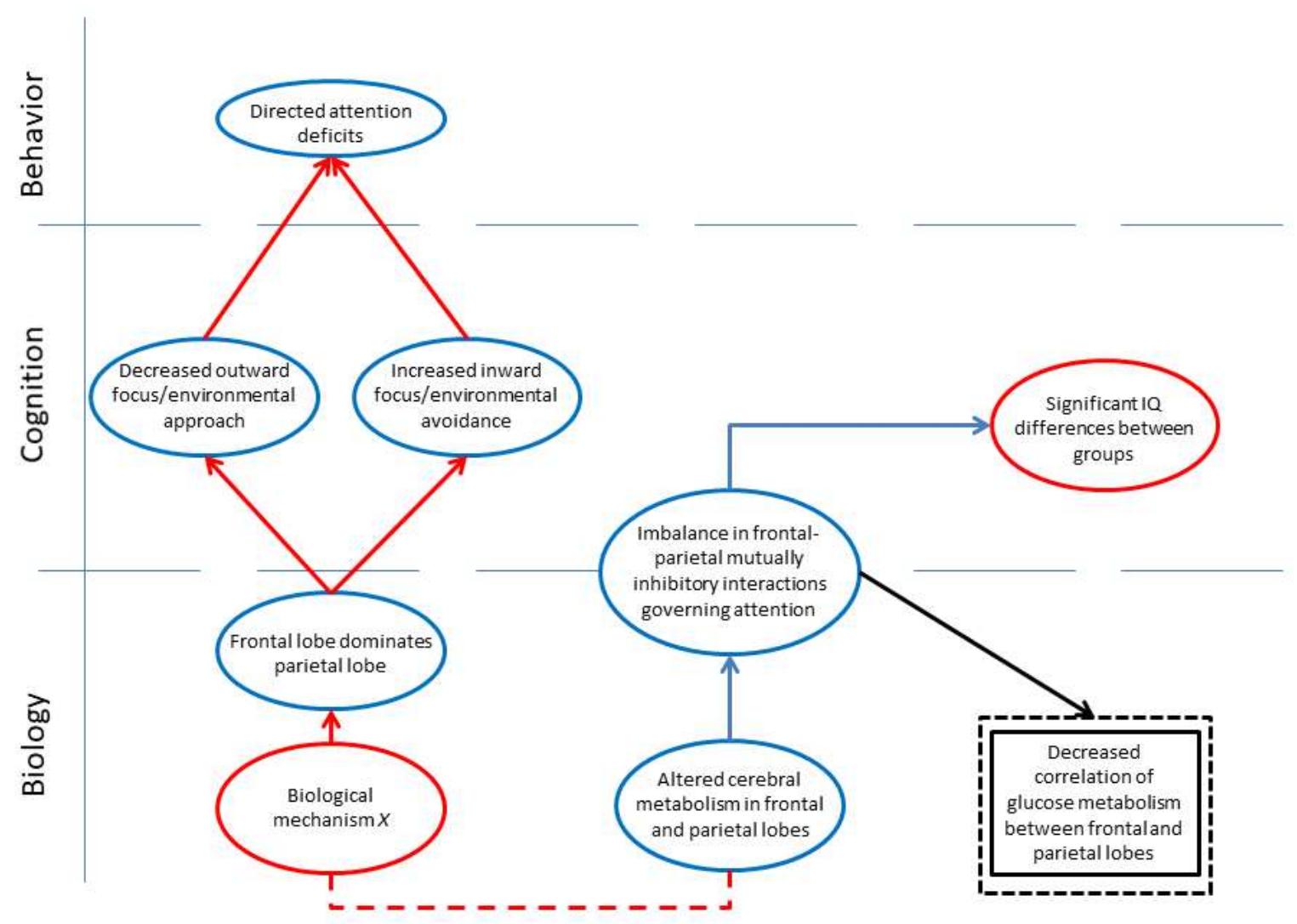

Figure 4. Alternative causal model that accounts for the results in Horwitz et al. (1988). Red lines indicate causal influences that are alternative to those proposed by Horwitz and coauthors. Biological mechanism $X$ refers to an unknown biological factor that is causally responsible for the ASD phenotype, and the dashed line linking it with altered cerebral metabolism in frontal and parietal lobes indicates the mechanism of correlation between the pathogenic biological factors of ASD and the pathogenic biological factors that lead to IQ differences, such as a common causal factor upstream of both.

The a posteriori hypothesizing by Horwitz and colleagues was no doubt helpful in establishing the plausible relevance of his findings for readers. The explication of a causal hypothesis, while necessary for the advance of explanatory science, may not currently be necessary to persuade reviewers, editors and readers of the relevance of observations, as the value of more descriptive data in the ASD-connectivity story is widely assumed. Although Horowitz's et al. observation 
has been well cited and set off a branching tree of ASD-connectivity work, the model they proposed has not been, to our knowledge, explicitly refined further.

\subsubsection{Early Structural Connectivity Observations}

These PET findings were not the only observation that set off the range of studies that have coalesced under the ASD-connectivity rubric. Courchesne and colleagues [47] used structural MRI to study brain development in ASD and controls. They examined children from the ages of 2-16 years, and found that children with ASD had larger brain volumes than controls over the first 2-4 years of life, but had substantially lower growth rates after age 4, such that by ages 1216 their brains were smaller in volume than those of the control group. This pattern of early overgrowth followed by subsequent slowed development was also observed in the white matter of the ASD group. Based on their findings, Courchesne and colleagues hypothesized a posteriori how the altered developmental course of white matter could have a causal role in the deficits in ASD. In typical development, white matter develops and matures under the guidance of interactions with the environment to create a brain optimally wired for daily function. In ASD however, they hypothesized that the behavioral phenotype of ASD may occur because of white matter maturation that occurs abnormally early and without the aid of developmentally appropriate interaction with the external world.

Two additional structural connectivity papers, both from Herbert and colleagues [48, 49], also found group differences in white matter volumes. Whereas Horwitz and colleagues (1988) speculated on the role of frontal-parietal connections, and Courchesne and colleagues (2001)

were generally silent on the matter of localization, Herbert and colleagues took an explicitly antilocalizationist approach, noting that the core features of ASD involved higher-order tasks that involved "cross-modal information processing." Citing connectionism [50]-a neural-network approach to studying cognitive function, whose neurobiological offshoots tend to minimize the role of localization in cerebral function-Herbert and colleagues suggest that the higher-order features of ASD result from distributed rather than localized abnormalities. This perspective presaged an influential FC paper in ASD. 


\subsubsection{Just's et al. Underconnectivity Theory (2004)}

Just and colleagues' initial foray into ASD connectivity [51] was a hypothesis-driven attempt to explain a particular cognitive phenomenon in ASD, and not the core ASD symptoms themselves. Individuals with ASD tend to show deficits in some cognitive abilities, but preservation or even enhancement of other, related abilities. Just and colleagues were interested in the dissociation between components of reading specifically: the enhanced ability of individuals with ASD to process the meaning of individual words coupled with a decreased ability to semantically integrate full sentences. They therefore predicted greater-than-typical BOLD activation in Wernicke's area (responsible for the local aspects of sentence processing), and less-than-typical BOLD activation in Broca's area (responsible for the global aspects of sentence processing) during a sentence comprehension task. Consistent with predictions, the ASD group showed greater activation in Wernicke's area than in Broca's area.

In addition to their hypotheses about Broca's and Wernicke's areas, they hypothesized that the observed deficits of sentence integration could be due to disrupted synchronization between distant cortical processing centers; this theoretical perspective was based on attempts of a behavioral neurologist [52] to explain "why patients with localized lesions sometimes displayed non-localized deficits"5.

Just and colleagues predicted decreased correlation in BOLD activation between spatially distinct regions. They in fact found reduced correlations of the time series of BOLD activation throughout ROIs of language regions in the ASD group. Combined with a reference to yet-to-bepublished data from their group on the Tower of London task (TOL; later discussed in conjunction with Just et al. (2007) and Just et al. (2012)), they suggested that FC differences likely existed in multiple task-related networks in ASD. This led to the first formulation of what they refer to as the Underconnectivity Theory of ASD. (We refer to this as Just's et al. Underconnectivity Theory [JUT], to separate it from other connectivity accounts of ASD. We specify JUT (2004) to contrast with JUT (2012), which follows in the next section.)

\footnotetext{
${ }^{5}$ It seems to us that Luria [53] , highlighted a gap in the explanatory power of localization rather than propose a solution, as did Mesulam [52] .
} 
JUT (2004), then, hypothesized that several deficits typical of ASD have in common that they all are dependent on communication between regions (Fig. 5). Social function, they posited, is a task that requires integration of disparate forms of information, which in turn depends on information transfer between distant regions of the brain. Executive function, and strategy shifting in particular, requires influence from prefrontal regions to other regions. Novel task learning requires dynamic reconfiguration of networks spanning multiple regions. The strength of the claim that social function is altered in ASD due to alterations in long-range cerebral connectivity is dependent at least in part on the proposition that intact social functioning relies on intact cerebral information integration, and the basic science evidence for this claim is (to our knowledge) yet rather scant.

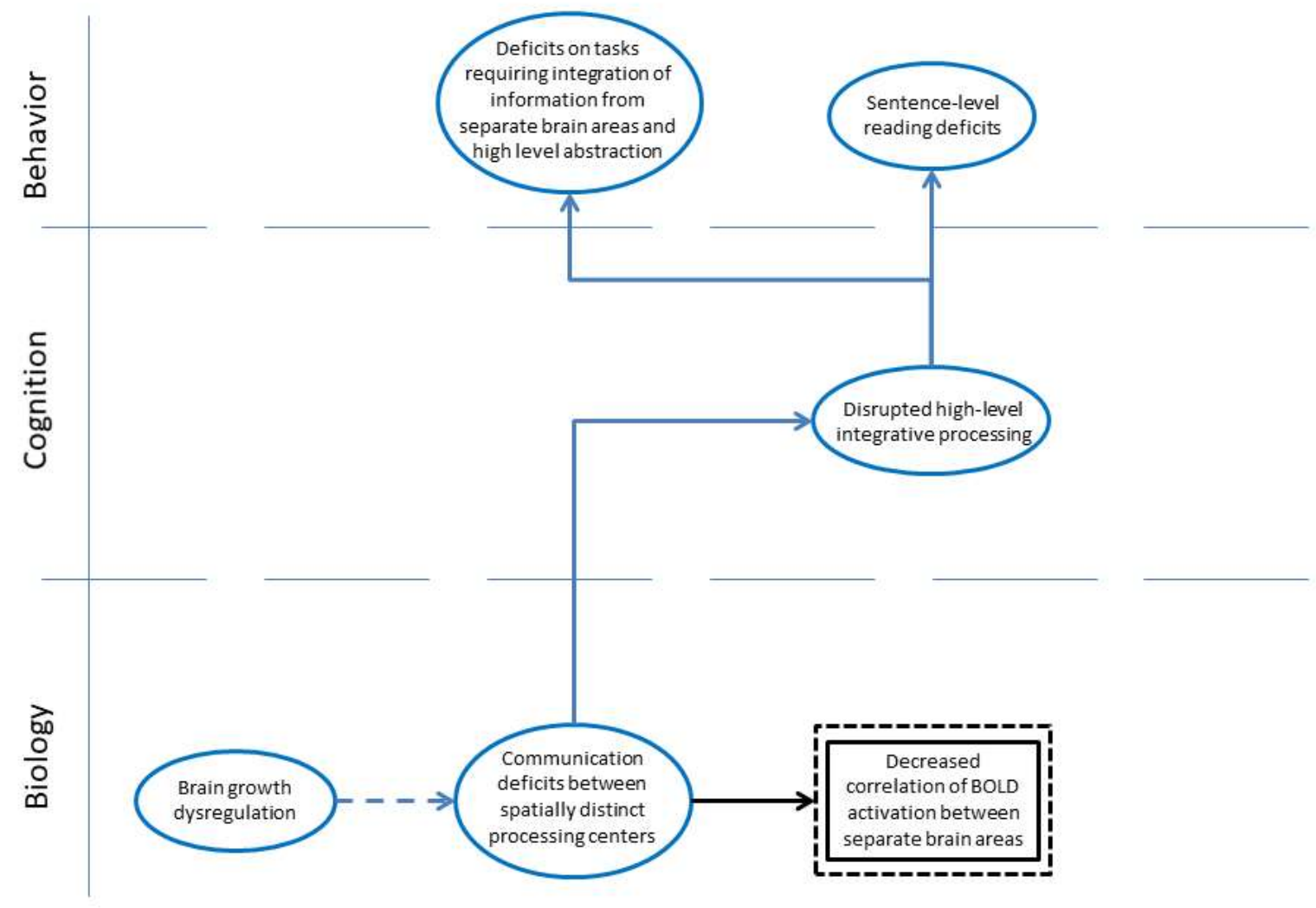

Figure 5. Causal diagram for Just's et al. Underconnectivity Theory of Autism (2004). 


\subsubsection{Belmonte and Local Connectivity}

We now take an important detour into local connectivity. Most of the work covered up to this point, as well as subsequently, focuses on long-range connectivity, i.e., the connectivity that occurs between macroscopically distinct brain regions. However, readers of the ASD connectivity literature will certainly have heard the mantra, "local overconnectivity and longrange underconnectivity." Careful readers of the literature will have noted that various papers have referred to "local connectivity" in the context of neurological constructs from level of minicolumns $(\sim 50 \mu \mathrm{m})$ to the space between EEG electrodes (multiple centimeters). In the case of Picci and colleague's review [11], "local" was taken as "characterize[d]... as the authors of the paper do"; a reproducible approach but not one which gets closer to synthesizing data about distinct well-defined candidate mechanisms. These spatial scales have, biologically, very little in common, and the consequence of futilely attempting to reconcile and synthesize data about such biologically disparate spatial scales is obvious. The only similarity is that different investigators have used the same term to refer to them: a difference without a distinction.

While the term "local overconnectivity" has subsequently become meaningless because of the many meanings assigned to it, Belmonte, Cook and colleagues [54] were originally concerned with connectivity within "neural assemblies"; this work follows on the observation of increased rates of epilepsy in ASD, Casanova's [55] finding of altered minicolumn structure, and Rubenstein and Merzinich's [56] proposal of altered excitatory-inhibitory balance. Belmonte, Cook et al. [54] is a masterwork that synthesized an immense amount of existing data from multiple levels of analysis, from genes to behavior. The mechanism at the core of Belmonte, Cook and colleagues' causal model was a reduced signal-to-noise ratio (SNR) in the autistic brain. First, they noted that a reduced SNR could manifest in two different ways, either as a neural system that contains so much noise the signal becomes undetectable, or a system with such weak signal it gets lost in the noise. They hypothesized that a system which could not adequately distinguish signal from noise would create an all-on or all-off sensory processing style, leading to an over-flooded, under-discriminant sensory processing system. Consequently, this overflowing stream of sensory information, not properly culled by early attentional processes, would overload early developing high-level processes. Since eliminating unwanted stimuli at a late stage in the attentional process incurs a higher cost than doing so at an early 
stage in the process, neurological computations which required the moderation of low-level processes by high-level processes would become much less efficient. Belmonte, Cook and colleagues predicted that the increased cost of computations involving high-level processes (rather than lower level ones) would lead to individuals with ASD compensating by relying more heavily on those processes which involved only low-level computations, and avoiding processes requiring high-level moderation whenever possible. This emphasis on low-level processes occurring in single neural assemblies, they said, would ultimately lead to the behavioral symptoms of over-focus on small details at the cost of the larger, similar to the view of Just and colleagues $(2004)^{6}$. The later Intense World Theory [59] extended the implications of this framework to propose a causal link to a variety of symptoms of ASD (Fig. 6).

The current state of the "local overconnectivity" hypothesis is somewhat uncertain. (See section 1.3.4.) The work of Butler et al. [60] has gone a long way in refuting the "neural noise" or "neural unreliability" hypothesis, which is, to a large degree, isomorphic to Belmonte, Cook et al. (2004), at least for the physiology measured by scalp EEG. However, strands of Belmonte, Cook et al. persist in the study of GABAergic mechanisms [61], which are not only increasing but serve as one of the theories that is semi-competitive (or yet-to-be-integrated) with the longrange underconnectivity hypothesis.

The second paper from Belmonte in 2004 (Belmonte, Allen et al. [62]) has set the motto, perhaps unintentionally, for much of the connectivity research that has followed: local overconnectivity and long-range underconnectivity. The paper itself was largely a brief synopsis

\footnotetext{
${ }^{6}$ Readers of the ASD-perception and ASD-connectivity literatures will recognize that more efficient processing of small, local features of stimuli accompanying decreased efficiency of global or gestalt percepts is commonly referred to as Weak Central Coherence (WCC) [57]. The line of thinking that resulted in WCC date at least to Rimland [41] . WCC is referred to in perhaps the plurality of the foundational ASD-connectivity literature as the psychological "target" of explanation, and is a term, if not a construct, that is largely specific to the ASD field (Belmonte, personal communication, 2019). Given its widespread use in the ASD-connectivity literature, why do we take pains not to call WCC by name? First, WCC is less of a theory than a descriptive analogy. Second, WCC applies to a perceptual phenomenon of ASD, and its explanatory scope does not include the core features of the condition. Third, the evidence that global perception within a modality and featural channel depends on longrange connectivity of the type that is at the heart of the ASD-connectivity literature is not clear to us. Finally, shortly after the publication of WCC in the first edition of Frith's book [57] , evidence from Happé began to undercut it-a point noted by Frith already in the second edition of her book [54]. Subsequent work by Mottron [58] has also provided alternatives to WCC. In the critical spirit of this chapter, we do not think it is productive, except in a historical perspective, to focus on WCC.
} 
of the contemporary literature and primarily a juxtaposition of the local overconnectivity of Belmonte, Cook et al. with the global underconnectivity of Just et al. [51]: “...in the autistic brain, high local connectivity may develop in tandem with low long-range connectivity."

However, prior and subsequent manuscripts made causal claims about the relationship between the local and the long-range. It is important to bear in mind that what was termed "local overconnectivity" by Belmonte, Cook and colleagues may manifest as "local activation" by the spatial resolution of fMRI tools, presaging Just and colleagues' [63] subsequent claim that local over-activity is caused by long-range underconnectivity (see below).

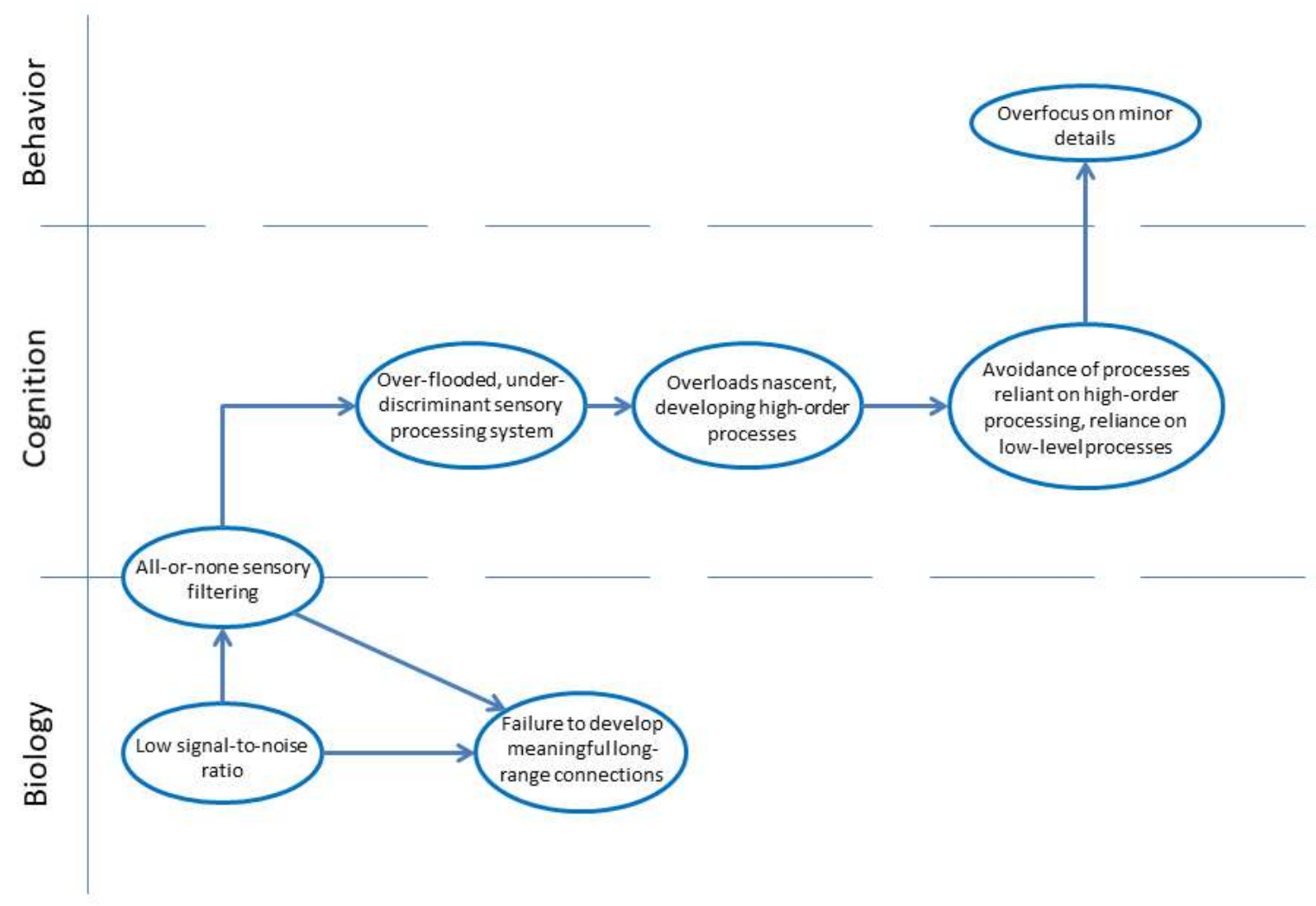

Figure 6. Belmonte's, Cook's et al. (2004) Causal Model Relating to Local Overconnectivity. 


\subsubsection{Just's et al Underconnectivity Theory (2012)}

Returning to long-range connectivity, in 2012, Just and colleagues revisited JUT with additional data [63] (hereafter, JUT (2012)) — specifically, the TOL data previewed in Just et al. (2004) and reported in 2007 [64]. TOL is a classical test of planning, which falls under the broad rubric of executive function. Executive function and planning specifically are known areas of weakness in ASD [18, 65-67]. Because individuals with frontal lobe damage are commonly and classically known to have deficits in executive function, by analogy, this fact adds a more localizationist flavor to the JUT (2012) account of ASD.

The fMRI results during the TOL task showed decreased measures of FC between frontal and parietal regions in individuals with ASD [64]. The absence of group differences in error rates helped exclude the alternative explanation that any group differences in their fMRI results could be due to group differences in task performance.

The new model began at the level of structural connectivity: citing the work of Courchesne and Herbert [47-49, 68], Just et al. hypothesized that the combination of increased brain size and abnormal white matter maturation and neuronal myelination across the first 2-4 years of life may create frontal-posterior pathways with long-range connectivity deficits caused by reduced transmission bandwidth. These bandwidth constraints would decrease the amount of information that could be effectively transferred between the frontal lobes and other regions of the brain, decreasing the functional influence of the frontal lobes and leading individuals with ASD to avoid tasks requiring top-down moderation by the frontal lobes. With time and development, they hypothesized that this would cause the posterior brain regions to develop increased amounts of autonomy as a compensatory mechanism. Ultimately, this increased autonomy would lead to enhanced performance on tasks which did not require involvement of the frontal lobes, explaining why children with ASD had been observed to perform better than controls on certain low-level processing tasks [63].

Just and colleagues, very much to their credit, move past vague notions of (semantic) connectivity and examined a more nuanced parameter — bandwidth — that is not directly observable as a measurement from BOLD data (see 1.3.1). To investigate this more specific and indirectly assessed parameter, they used a generative computational model that was able to adjust 
this parameter. The computational model [69] generated simulated fMRI data that was then compared against recorded fMRI data [64] in individuals with ASD and controls. The model was run with three different permutations of parameters (and three resulting sets of simulated data), and that model which included both decreased frontal-parietal bandwidth and increased parietal autonomy best fit the observed fMRI data.

The revision of JUT (2004) into JUT (2012) involved a substantial leap into additional levels of analysis and causal claims (Fig. 7). It also fulfilled the promise of Belmonte, Allen et al. (2004), of specifying the causal relationship between local cortical function (overlapping with Belmonte's notion of "local connectivity") and long-range underconnectivity. Additionally, it specified explicitly which changes were pathogenic, and which were compensatory. As a part of this specification, JUT (2012) took on a developmental stance, specifying the consequences at $t_{1}$ of atypicalities at $t_{0}$, i.e., that increase autonomy in posterior regions was caused by decreased frontal-posterior communication. 


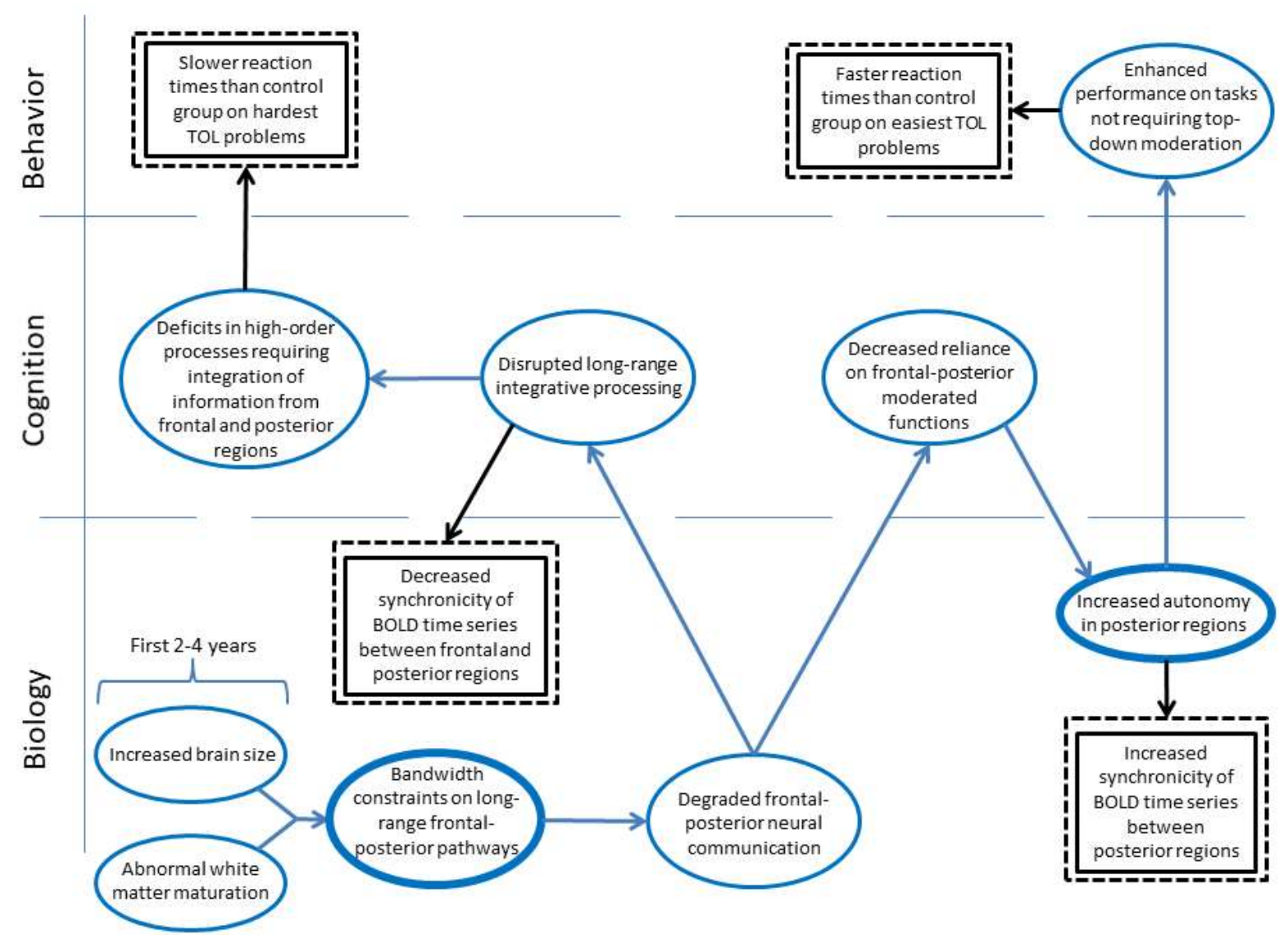

Figure 7: Causal diagram for Just's et al. Underconnectivity Theory (2012). TOL = Tower of London.

\subsubsection{High-Time-Resolution Synchronization Accounts}

The final family of explanatory models we present features temporal synchronization deficits between brain regions as a specific mediator of altered connectivity. The causal model we highlight in the figure is the Temporo-Spatial Processing Disorder (TSPD) hypothesis of ASD proposed by Gepner and Feron [70]. The motivating observations were that individuals with ASD had a decreased ability to perceive and integrate dynamic multisensory stimuli, and to produce online sensory-motor coupling and adequate verbal and nonverbal outputs. The core component of the TSPD model is an inability to properly temporally process and bind rapid stimuli, as is the core component of an additional two related models we consider. The proposed connectivity alteration resulting in TSPD is somewhat non-specific in terms of distinguishing it from other network-level accounts of ASD. Candidate systems neurobiological accounts include 
both cortical-cortical mechanisms, as in other connectivity accounts, as well as cerebellar and basal ganglionic mechanisms.

Altered temporally sensitive long-range synchronization is proposed to include consequences in auditory, visual, attentional and sensory-motor domains. In the auditory modality, Gepner and Feron hypothesized that an inability to correctly process rapid stimuli degrades the ability to perceive and process brief acoustic transitions (such as those present in normal conversation). This, in turn, leads to impaired development of speech and language production and comprehension. In the visual domain, temporal processing deficits lead to the inability to properly perceive rapid biological motion, which affects several behaviorally important processes. Individuals with ASD may be unable to adequately process eye movements (the fastest movement humans can make), leading to deficits in eye contact and joint attention. Additionally, they would be unable to properly process lip movements, leading to impaired visuo-auditory perception and language comprehension, as well as emotional facial and body movements, leading to deficits in empathizing. Finally, they hypothesize that the inability to process faster parts of moving stimuli may lead individuals with ASD to over-focus on static elements of stimuli, potentially leading to enhanced local perceptual processing. Both the TSPD and the TBD theories go on to argue how low-level sensory processing deficits could be important causal contributors to the higher-level behavioral deficits present in the ASD phenotype (Fig. 8).

Related to TSPD is Brock and colleagues' Temporal Binding Deficit (TBD) hypothesis [71], which characterized the deficits in the ASD phenotype as the product of temporal binding impairments between separate components of networks involved in feature recognition. This hypothesis is explicitly based on a neocortical dynamics model by Nunez [72]. It also has a number of similarities to a distinct cortical function model developed by Uhlhaas and Singer, primarily to explain schizophrenia but also applied to ASD [73, 74]. Like several other early connectivity-related theories, Brock et al. were motivated by observations that children with ASD have difficulty integrating individual visual percepts into a gestalt.

Uhlhaas and Singer have proposed that synchronization also supports short-term memory, associative memory, sensory-motor coupling, decision-making, motor planning and consciousness [75]. As in other connectivity theories, they attributed these performance 
differences to long-distance connectivity alterations, but they further specified their connectivity construct as a temporally-sensitive binding mechanisms between local networks particularly involving the beta $(13-30 \mathrm{~Hz}$ ) and gamma bands (around 30-80 Hz). The soundness of this framework is challenged by unresolved questions as to whether perceptual binding, generally, is subserved by neural synchronization [75]. This point reinforces the general need for basic science (neurotypical) data to clarify causal mechanisms for the cognitive and behavioral features which are proposed to be altered in ASD.

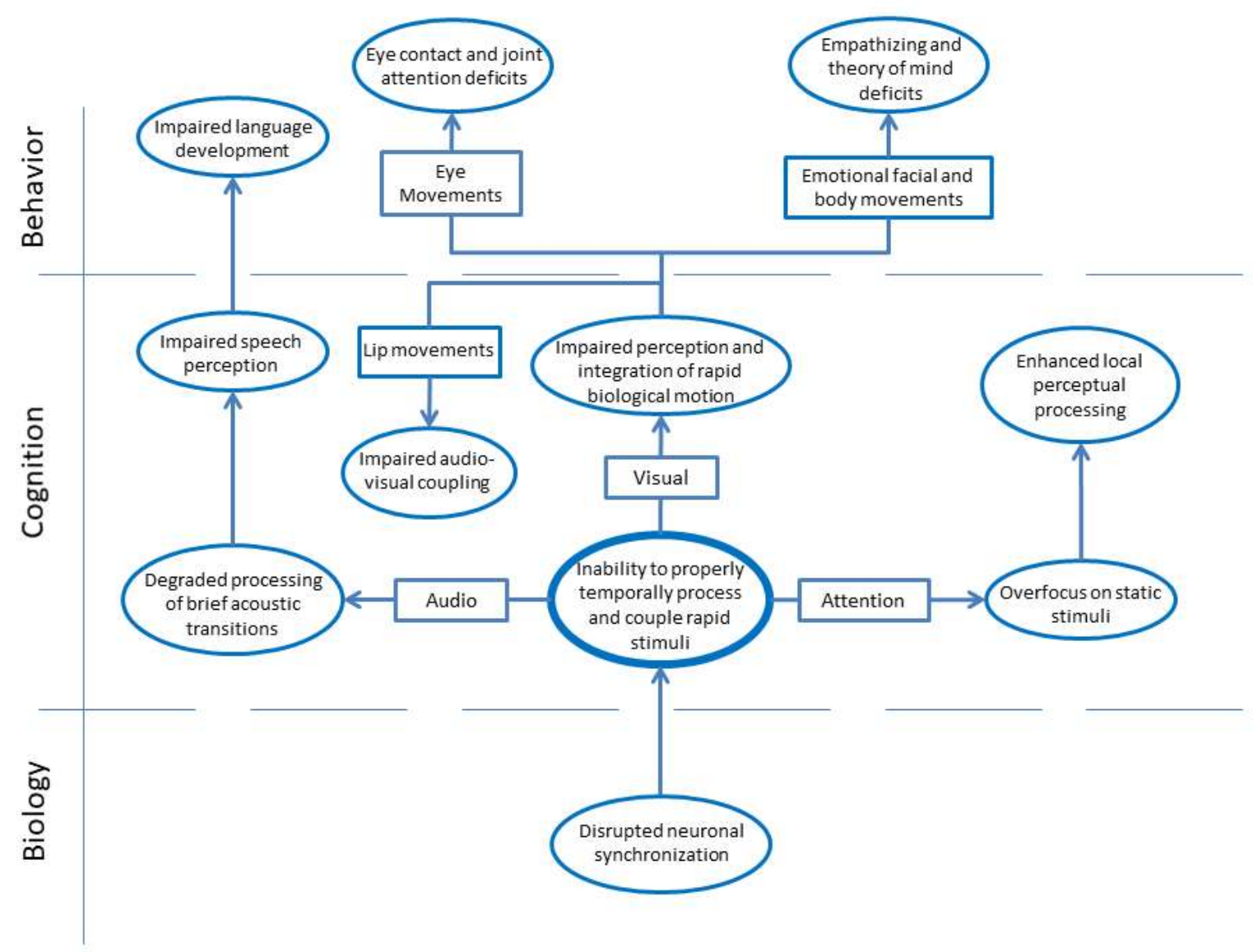

Figure 8. TSPD causal diagram

\subsubsection{Summary and First Attempts at Synthesis}

The theories presented above differ widely in behavioral explananda, neurobiological/cognitive constructs, imagining metrics of connectivity, fundamental views of brain function and the role of development [23] (Table 1). At first glance, given so many variables, the theoretical landscape 
seems relatively sparsely populated, which makes it difficult to construct either/or empiric tests to falsify one account and support the other. The problem of falsification is further complicated by the amount of activity going on in the brain that is hidden from current methods. It may appear that disproving " $\infty-1$ " alternative hypotheses is a non-starter. However, we can already identify places where our even sparsely populated accounts come into (testable) contact with each other. The nature and identity of the networks that are relevant to ASD differ by theory. JUT (2012) [63] specifically privileged connections between frontal and posterior regions in the genesis of ASD. By contrast, JUT (2004) [51] used the example of reading comprehension to make the general point that activation of more focal brain regions serve the more basic corresponding functions (e.g., word comprehension) and spatially broader networks subserve more complex functions (e.g., sentence comprehension) which may be differentially affected in ASD. The implication in JUT (2012) is that the range of the ASD phenotype is a function of impairments in abilities that depend on frontal-posterior communication, whereas the implication of JUT (2004) is that the ASD phenotype is reflective of impairment in "higher-order" abilities requiring connectivity between brain regions and preservation/enhancement of related abilities that require only regional activation.

A second area of direct contact between models is in the direction of influence between local neural activity and long-range connectivity. JUT (2012) [63] claimed that local over-activity is caused by long-range underconnectivity, whereas Belmonte, Cook Herbert and colleagues [49] flipped the causal arrow, claiming that white matter alterations in ASD are due to abnormal neural activity/neural noise. (Oligodendrocytes, which myelinate the central nervous system, are responsive to neural activity). We might make progress in disambiguating the direction of the arrow if we had longitudinal physiological data, an agreed-upon metric of relevant neural noise and a causal approach to statistical modeling [76].

Finally, we note that, while many authors are non-specific about what they mean by “connectivity," several have already specified their constructs. JUT (2012) [63] studied bandwidth; Belmonte, Cook et al. [54] studied SNR; and the models in section 1.2.6 studied temporally sensitive synchronization. In the following section, we develop these concepts further. 


\subsection{Specificity in the Construct of Connectivity}

The goal of this section is to increase specificity in our brain-theoretical constructs around connectivity. Before proposing these constructs, we first wanted to highlight where task-related data can aid us in our enterprise. Task-related data independently controls psychological variables and while using imaging as the dependent variable [7]. This approach limits the probability of falsely affirming a cognitive-imaging link when the brain state is not controlledso-called "reverse inference" [77]. However, the problem of reverse inference is not generally relevant to the ASD-connectivity literature, where the inference typical does not proceed from imaging data alone to cognitive consequences.

Where task-related data can be useful is in helping to refine a causal graph by ordering the elements based on data rather than theory alone. The physiology that drives the behavior in a task may not be the same as the physiology that is statistically associated with the diagnosis, particularly when a non-diagnostic behavioral explanandum is used. In such cases, a performance confound could lead to confusion about whether group differences in physiology are primarily related to behavioral performance or to diagnosis. McAuliffe et al., in an EEG physiological study of motor behavior in ADHD, used moderation analyses to distinguish where the effect of diagnosis was upstream of the EEG physiology that correlated with task performance vs. where diagnosis moderated the link between physiology and behavior [78].

Task-related data are also critical to phenomena that are specific to task-related modulation (see section 1.3). Finally, task-related data are critical to testing many of the constructs we propose in the following sub-sections.

Models adopt the assumptions of their "parent" brain and cognitive theories, as described above in the discussion of localizationist vs. holist brain theories. Here, we use an information theoretical framework, a sort of electronic-engineering analogy of brain function.

We present some of the strongest literature that has used these terms in cognitive neuroscience. There is some variability in the use of the terminology, which is a function of the fact that these words are conventionally used to mean different constructs in different fields. The constructs 
presented are not fully independent, and the set is certainly not exhaustive. Further, the metrics that we propose have not been validated as true measures of these constructs.

Our thrust, then, is to offer these constructs as semi-distinct and contrasting, in order to start a conversation about how to increase specificity within the macro-neuroscience construct of connectivity, both generally and in ASD research.

\subsubsection{Bandwidth}

Based in the Shannon-Hartley theorem and Information Theory, bandwidth is defined as the transmission capacity of a communication channel, taking into account the impact of noise [79] ${ }^{7}$. In our context, bandwidth refers to the maximum amount of information that can be transmitted between brain regions per unit time [63]. Several factors can impact bandwidth, including transmission speed [80] and noise [63, 79], both described in greater detail, below.

Several studies $[81,82]$ indicate that a limited semantic bandwidth may be one possible reason for cognitive capacity limitations (e.g., in attention, working memory). As above, we contrast semantic information with a physical property (viz., entropy) of the physiological signals that we record with fMRI, EEG and other techniques. This latter sense of information is trivial to calculate from the signals, but we are only at the starting point of how to link information/entropy measures of the physiological signals with the semantic information that is functionally (behaviorally) relevant.

We can see three approaches to bridging functional brain imaging with the latent semantic information: using a cognitively based computational model which generates simulated imaging data and whose output can be tested against actual imaging data; strengthening our understanding of how semantic information is coded within the signals we can measure; and performing psychophysical tasks in which the information input is controlled, and information output is measured such that the internal structure of the "black box" brain can be inferred from the transfer between the two.

\footnotetext{
${ }^{7}$ Note that "bandwidth" in the connectivity literature can also refer to the frequency range of recorded physiological signals. That is not the sense we are using here.
} 
A compelling example of the first approach is JUT (2012) (section 1.2.5). As discussed extensively in the previous section, Just and colleagues used a 4CAPS generative computational model that allowed the investigators to vary bandwidth parameters. The various "runs" of the model, using these different values of the bandwidth parameter, then generated simulated imaging data, against which actual, observed data were compared.

Concretely, the 4CAPS computational model imposes a bandwidth constraint by limiting the amount of activation that representational elements communicating between the executive (frontal) and spatial (parietal) centers can consume at any time. There were no explicitly transmitted or received elements in this implementation; instead, the representation elements were placed in the communication channel by one center so that other centers could access them. The bandwidth constraint in the model effectively limited the throughput of communication channels containing representational elements generated by the execution center or spatial center for use by the other centers. This constraint limited the speed of executive-spatial communication, stimulating the hypothesized alteration of connectivity between the frontal and posterior regions.

In addition to the bandwidth constraint implemented in this way, bandwidth limitations can also be simulated by adding Gaussian white noise into the model. Due to the disturbance of information transmission imposed by the noise, the amount of valid information received may be reduced, therefore effectively decreasing the bandwidth.

A second proposed approach for assessing the effect of bandwidth limitations is to measure the entropy (physical information) within physiological signals, and to interpret this information in light of simultaneous task performance. There is a nascent body of research looking into how these measurements correspond to semantic information. Mutual Information (MI) is a measure based on Information Theory and examines informational dependence between two signals; it is, in a sense, a generalized form of correlation and is a measure of how much is learned about the distribution of one signal by observing another. The higher the value of MI, the more information transferred between two systems within a time period, and hence the greater the bandwidth. 
MI is nonlinear and is more suitable than linear approaches ${ }^{8}$ for analyzing and processing nonstationary signals; it can better describe the behavior of complex systems like the human brain. MI reflects bandwidth of the observed time series and does not necessarily correspond to the bandwidth of semantic information flowing through the mind. The hopeful news is that the field is beginning to unravel how local field potentials (LFP) encode functionally relevant (semantic) information [83]. As LFPs represent the "micro-scale" signals that contribute to the "macroscale" EEG, an accumulation of knowledge at LFP and scalp EEG levels (with penetrating and surface ECoG measurements intermediately between the two), measuring MI under multiple different task conditions, could help us eventually derive specific conclusions about semantic information bandwidth based on scalp EEG recordings. We can also envision how this enterprise could be helped by machine-learning decoding approaches which are able to predict trial-by-trial perception or performance. Such methods can sometimes help "localize," in whatever parameter space (e.g., frequency, topography), those components which most contribute to correct classification [84]. Moreover, classification performance could reveal the minimal information content of the signal. This would help us focus our subsequent search for mechanistic interpretations, using parametric analysis tools.

The final method we propose is not exclusively related to bandwidth but seems to fit in this section as well as any other. The goal is to infer semantic information transfer within the brain by controlling the signal and noise content of input sensory information and task procedures in such a way that the resulting behavioral output can be interpreted to "reverse engineer" the information flows and transformations. Actual physiological brain data need not necessarily be collected, particularly if the brain architecture of the relevant sensory and motor systems is well understood. Multi-sensory tasks may fit this bill [85].

An example of this approach in ASD comes from studying the differential effect of perceptual error within the somatosensory system (proprioception) vs. the visual system on motor output. The laboratories of Mostofsky and Shadmehr used a task in which children with ASD and

\footnotetext{
${ }^{8}$ We have not considered in great depth whether linear or non-linear approaches are most appropriate for studying brain physiology. The authors are not experts in this area but have colleagues and collaborators who have strong (and opposing) views on the subject. To summarize, some argue that the brain is a non-linear system and therefore must be modeled/analyzed by non-linear techniques. Others argue that, while the brain is indeed a nonlinear system, it is estimated quite well by linear techniques, which offer far more analytical flexibility.
} 
controls had to push a manipulandum toward a target, while proprioceptive and visual input were systematically rotated [86, 87]. Compared with controls, children with ASD showed oversensitivity to proprioceptive error and under-sensitivity (relative to controls) to visual error. No brain data were collected, yet the investigators were able to measure how changing different types of information - from distant locations in the brain-impacted behavior.

\subsubsection{Transmission speed}

Transmission speed is highly related to bandwidth, via the Shannon-Hartley and Nyquist theorems; it can be thought of as the speed of fluid through a pipe, whereas bandwidth is akin to gallons per hour. The higher the transmission speed, the greater the bandwidth, all else being equal.

While individuals with ASD do not have gross white matter and myelination abnormalities, as do patients with multiple sclerosis, for example, subtle changes have been noted in ASD, and their relationship to FC has been suggested. Diffusion Tensor Imaging (DTI) studies repeatedly demonstrate group differences in white matter [88, 89].

However, white matter differences only set the stage for-yet do not by themselves proveissues with transmission speed. For measuring conduction speed, the best approach may be to use time-domain measures from high-time-resolution modalities, such as MEG and EEG. Eventrelated potentials (ERPs) are responses that are time-locked (and phase-locked) either to a task stimulus or response. Averaging in the time domain reveals these signals, which are about halfan-order-of-magnitude smaller than the spontaneous M/EEG signal. The speed of conduction is typically measured by the latency of the relevant waveform ${ }^{9}$ (Fig. 9).

\footnotetext{
${ }^{9}$ Note that, in a peripheral nerve, demyelination tends to affect some axons more than others. Rather than a change in onset latency, clinical nerve conduction velocities in the presence of demyelination tend to show temporal dispersion: a widening of the wave and decrease in the peak amplitude. We are unaware whether the situation is analogous in the CNS.
} 

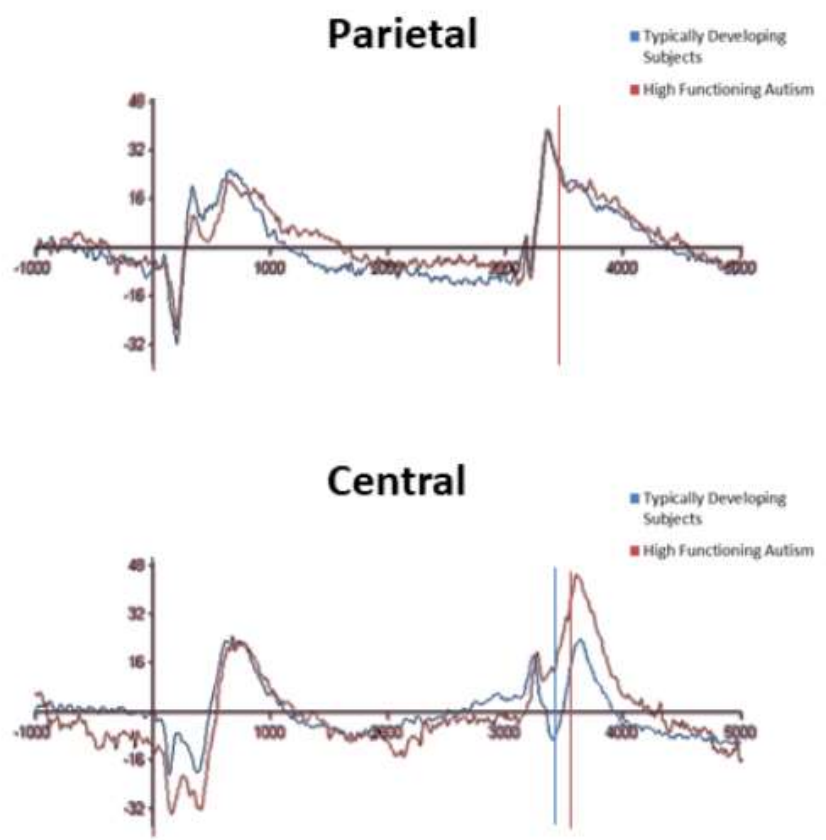

Figure 9. Use of Time-Domain Analyses from Two Scalp Sites to Assess Transmission Speed. In data from this conference abstract [90], event-related potentials is measured during a praxis task, to assess timing of cortical responses understood to flow from parietal to central regions. The activity of interest occurs starting around 3000 msec. Children in both groups have activity with similar timing in the parietal regions, but children with ASD (red) have a slower response in downstream central regions, compared with typically developing controls (blue).

Many connectivity accounts of ASD focus on cortical-cortical transmission (or corticalsubcortical-cortical transmission), and it is possible to measure the differential onset latencies of the ERP waveform from known regions within a functional, neuropsychological network [91], though this approach is not often taken. The best characterized account of slowed transmission in ASD, however, is within the early stages of the auditory system, starting from the ear. Roberts' laboratory [92] first examined the latency of early auditory ERPs in an investigation of plausible auditory abnormalities as underlying altered language development as well as auditory hypo/hyper-sensitivity. Over an accumulation of work, they have found children with ASD had an 
average of $11 \mathrm{msec}$ latency delay in the auditory M100 at right superior temporal gyrus, and in fact with high classification accuracy [93]. Children with ASD and little or no spoken language had an average latency delay of $18 \mathrm{msec}$ [94].

Compared with most potential "rules of correspondence" between levels of analysis, the bridge between transmission speed (physiology) and structural connectivity (anatomy) is thought to be relatively well understood. Transmission speed depends on myelination and on synaptic transmission. Our understanding of these associations benefitted from more accessible and parallel processes in the peripheral nervous system. (The relationship between transmission speed and more subtle structural phenomena, such as differences in "white matter integrity," as indicated by alterations in DTI measures, is perhaps less well understood.)

Recent work from Roberts' group has begun to combine transmission speed measurements (evoked-response latencies) with DTI measures, in order to bridge these levels of analysis. They further incorporate magnetic resonance spectroscopy (MRS) measures of GABA levels as a proxy of synaptic transmission speed; this inclusion further serves to bridge the connectivity account of ASD with a GABAergic account $[61,95]$. They show [96] that children with lownormal fractional anisotropy (FA; "white matter integrity") have M50 auditory event-related field (ERF) components predicted by GABA levels (and therefore presumably synaptic transmission), whereas those with yet lower FA values have a correlation between M50 latency and FA values, implicating white matter integrity (Fig. 10). 


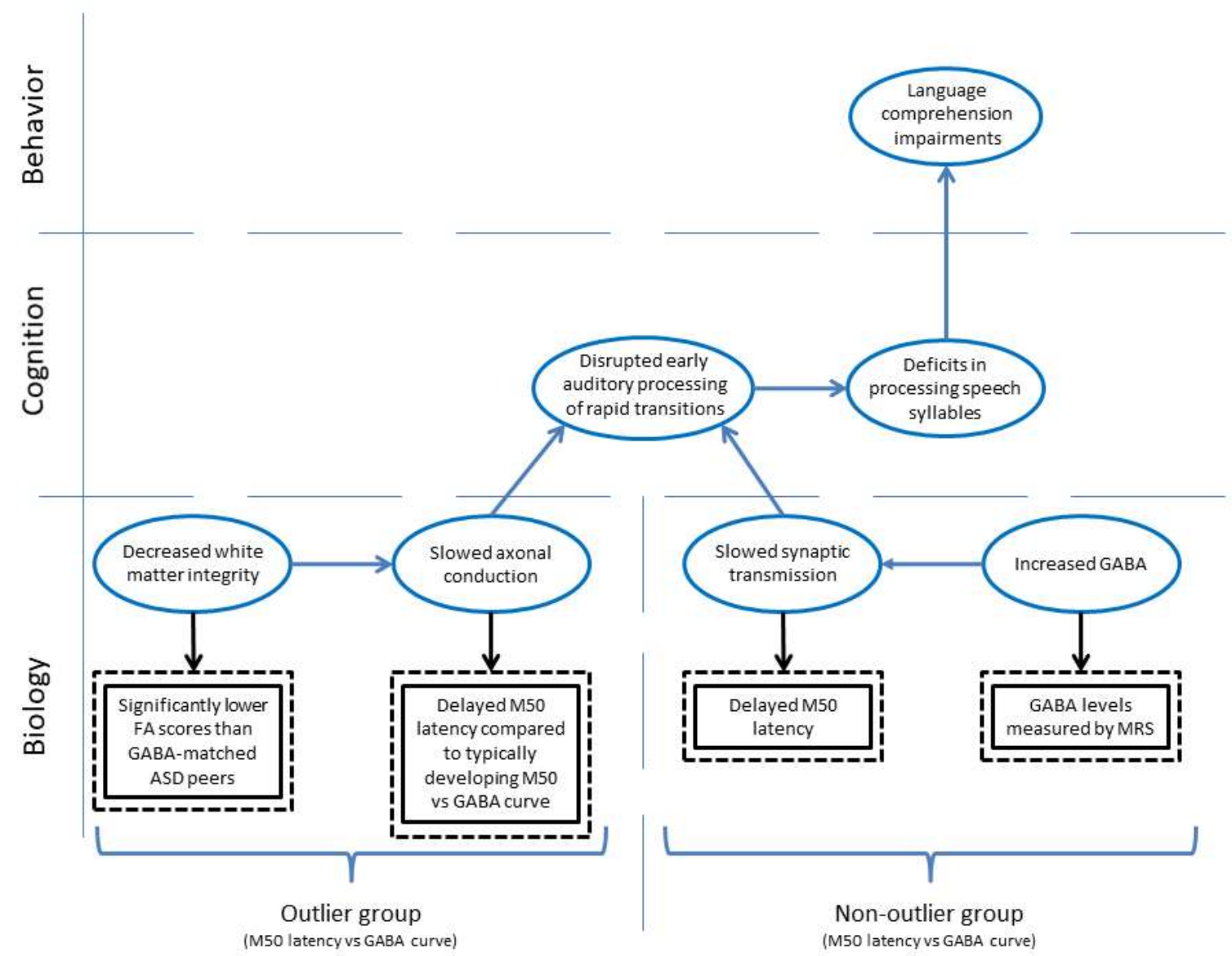

Figure 10. Causal Diagram representing Roberts' et al. Conference Abstract on Transmission Speed in ASD.

EEG and MEG are the techniques that have the temporal resolution to address questions of transmission speed. However, with EEG in particular, volume conduction represents a potential confound to contrasting measurements from two sensors. Volume conduction artefactually transmits signal from one brain generator to a sensor overlying another generator. Volume conduction affects not only the proposed measurement of transmission speed but any simultaneous comparison of the physiology of two or more regions as well (e.g., synchronization, below).

Because of volume conduction, signals in the EEG sensor space may exhibit spurious connectivity [97]. Synchronization can be affected by volume conduction; i.e., lead-field operator of EEG time series is a spatial low-pass filter, so the activity of a single-point source generates temporally synchronized dynamics in EEG recordings. As a result, the synchronization 
observed may be a mixture of true synchronization and the spurious synchronization caused by volume conduction. Several methods have been proposed to overcome the volume conduction effects in scalp EEG connectivity analysis. For example, researchers use a spatial filter (e.g., Laplacian montages), and source localization methodologies have been proven to be a useful way of overcoming volume conduction and computing EEG source connectivity [98, 99]. Some investigators are anecdotally concerned that the investigator-selected choice of analysis parameters may have a material impact on the results, but sensitivity analyses may be used to mitigate these concerns. Previous studies [100] have proposed that EEG source connectivity is relatively unaffected by the choice of forward model and anatomical templates, whereas different inverse methods induce different connectivity results. The beamformer is a technique that estimates a voltage- $v s$.-time series from a particular region in source space $[101,102]$ using a minimum variance, distortionless response spatial filter. It can therefore be used with timedomain analysis methods, like ERP/Fs.

\subsubsection{Signal-to-noise}

"Noise" may be generally defined as signal variability that is not positively related to task performance. In this broad definition, we include instrument error and extra-cerebral artifact; in EEG particularly, types of noise include movement, muscle and eye movement artifact. Multiple methods and pipelines exist for detecting, rejecting and/or correcting this noise in EEG, MEG and fMRI, however optimization and validation of these techniques suffers from the fact that there is no "ground truth" to say what the signal should look like, absent the artifact.

What we refer to here, however, is "neural noise," i.e., noise contained within the computational system of the brain. However, even if we had a cerebral signal that was collected with perfect fidelity, it would be impossible, without behavioral context, to characterize what is signal and what is noise. In this sense, complexity (entropy) that enhances performance would be characterized as signal, and variance that is associated with a decrement in performance would be noise.

Neural noise, as generally construed, may not always necessarily be a bad thing. Some amount of neural noise may be important for optimal functioning [103-105]. Neural noise can increase 
the sensitivity to weak signals, a phenomenon known as "single resonance." Moreover, neural noise can be beneficial for faithful transmission of high frequency inputs [103]. However, excess noise can negatively impact bandwidth [63].

It is within these caveats that we consider neural noise in ASD. The notion of noise is less directly related to long-range connectivity than to local connectivity, as originally proposed by Belmonte, Cook et al. (2004) and as refuted by Butler et al. [60]. Indeed, the family of neurobiological theories implicating altered inhibitory-excitatory balance or alteration of GABAergic function has been seen, at least partially, as a competitor to the connectivity theory. However, SNR is also an element of multiple explanatory models of connectivity (e.g., [48, 49]).

SNR has a long history in ASD research, exemplified by Belmonte, Cook and colleagues (2004), but also studied by others [95, 106-108]. However, the results of Butler and colleagues [60] go a long way toward refuting a "neural noise" or "neural unreliability" account of account of ASD, at least as far as the signals we can measure at the scalp. They used several methods to test the reliability of neural signals in autism. Firstly, they assessed ERPs. An unreliable signal may have broader and delayed ERP components in the individual-participant average waveform. Secondly, they examined the evoked power spectrum. The frequency decomposition of unreliable signals may have a lower pass-band. Thirdly, they looked at the consistency of phase. Greater variability in phase dispersion across trials may exist in unreliable signals. Fourthly, they observed variability of power. An unreliable signal may have greater variability in amplitude. The results showed no significant differences in any of the proposed measures of SNR between individuals with autism and neurotypical controls for either the visual or somatosensory responses.

Other studies have used different methods: Dinstein and colleagues [106] calculated SNR by dividing response amplitude by variance across trials; others have calculated the standard deviation of the entire ERP epoch $[109,110]$. Inter-trial coherence (ITC) is yet another method.

Despite these findings, other versions of the inhibition/excitation hypothesis [96] continue to be open lines of exploration, including via increasing study of GABAergic alterations in ASD [61]. SNR may be most useful as a candidate explanation of the attentional differences in ASD. Arazi and colleagues [111] found that visual cues that modulated alertness and spatial attention 
decreased trial-by-trial neural variability. These findings illustrate the role of attention in the modulation of signal-to-noise in the neural system, and the relationship between signal-to-noise and behaviors.

A significant concern to interpretation of SNR measures in ASD is that these methods are sensitive to non-physiological noise, and individuals with ASD may have an increased magnitude of this artifact, including muscle and eye-blink artifact in MEG/EEG $[112,113]$.

\subsubsection{Synchronization}

The term "synchronization" warrants explicit definition within the current context. In the most general sense, it refers to the strength of the temporal relationship of two events (either repeated, transient or oscillating/ongoing), either behaviorally or within the brain. Seeking an ostensive definition by examining the ASD-specific reviews of "synchronization" by field-leaders Uhlhaas and Singer $[73,74,114,115]$, one finds references to both local induced oscillatory activity ${ }^{10}$ and long-range coherence of slow (fMRI) physiological time series [51]. Because both fMRI and EEG measure primarily oscillatory activity, and because most measures we call "connectivity" look at the temporal similarity of two physiological time series, this broad definition of "synchronization" may admit most concepts referred to under the rubric of FC"11.

The specific behavioral phenomenon being explained by Brock and colleagues [71] as well as Gepner and Feron [70] is somewhat more restricted. Both accounts focus on the temporallysensitive integration of brief stimuli; this focus on high-resolution temporal coordination is how we use the term synchronization. Extended accounts of how synchronization (in this sense) supports brain function include the integration or binding of motor plans and of remembered information. So while both EEG and fMRI measure ongoing oscillatory activity, the important consideration is how transient information is encoded on that carrier wave, with high temporal precision, and whether that temporal precision is aberrant in ASD. To achieve this, we need

\footnotetext{
${ }^{10}$ Consistent with the notions of "local connectivity" in Belmonte, Cook et al. (2004), if possibly evidence against his hypothesis [116] [117] -local "undersynchronization" rather than "overconnectivity"

${ }^{11}$ We do not dispute that Uhlhaas and Singer's inclusion of data is consistent with their model of synchronization; rather, their model is quite inclusive relative to the concepts listed under the rubric of FC.
} 
information regarding how temporal information is encoded in MEG/EEG signals (fMRI likely does not have adequate temporal resolution) as well as analysis methods that have a temporal resolution adequate to parse apart responses to brief, transient stimuli.

Synchronization in physical systems is used to represent the relative relationship of two or more systems over time, including chaotic systems $[118,119]$. In the broadest sense, the wide variety of tools that measures synchronization, includes coherence, which is perhaps the most widely applied FC method in clinical EEG [120]. However, we again constrain our use of the term synchronization to apply to high-time-resolution changes in cortical activity and need to find methods that meet this high bar. Relevant synchronization phenomena include phase synchronization and generalized synchronization [121, 122]. While phase synchronization methods, including Phase Locking Value (PLV) [123], are widely used in EEG signal analysis, relatively long analysis windows can mean that the effective time resolution is inadequate to the task set forth by Brock and colleagues [71] and Gepner and Feron [70]. As the window length is defined by the number of required cycles rather than the absolute time, the measurement of highfrequency oscillations [75] may mollify this concern. However, this approach is only valid in the case of nearly periodic signals [121].

Generalized synchronization, by contrast, may be assessed without assumptions of stationarity (given potential transient activity induced by perceptual stimuli or motor response) and with the time resolution of a time-domain technique $[121,124]$. Generalized synchronization is computed by reconstructing the trajectories in the state space of the underlying dynamic system for each time series [125].

Stam and Dijk [121] proposed Synchronization Likelihood as a time-domain method suitable for analyzing non-stationary signals and has excellent temporal resolution. Moreover, Synchronization Likelihood is fairly robust even in noisy data. Data of individuals with ASD often contain considerable noise, including muscle artifact, motion artifact, eye blinks and eye movements.

Concerns about volume conduction are as relevant for the assessment of synchronization as they are for assessing transmission speed at multiple sites. Source localization and source 
separation methods are relevant, again with concern about how the investigator-set analysis parameters could affect the dependent variables.

\subsubsection{Task-Related Modulation}

The last construct we examine is the possibility that the alteration in ASD is not in connectivity per se, but in the mechanisms that modulate inter-areal communication in response to task. Pillai and colleagues [126] found that task-negative baseline measures of PLV did not differ between groups (ASD and control), but rather that modulation of the connectivity measurement variedin opposite directions - in response to the performance of a motor task. It may be that a mechanism or mechanisms unknown that modulate inter-area communication in response to a task are altered, rather than the mechanisms that permit that communication. This paper also proposed, via comparing clinical correlations to distance in connectivity measures from the control distribution, that targeted intra-hemispheric group differences in connectivity were pathogenic, whereas inter-hemispheric differences in connectivity were compensatory.

\subsection{Discussion}

1.4.1 Linking to the Broader Literature: Heterogeneity, Other Theories, Other Neuropsychiatric Conditions

Systematic reviews of the descriptive literature [9-12] generally support the notion that individuals with ASD show differences in connectivity metrics compared with peers, and nonparametric machine learning algorithms acting on fMRI connectivity data can correctly predict infants will be diagnosed with ASD [127]. While concerns about analysis pipelines, sample size and artifact still hold [39], large-scale studies [128] and methodological research [38] are attempting to address these concerns. The preponderance of results gives prima facie evidence that what is being measured by these analyses could be relevant to the pathogenesis of the behavior. 
Moving from description to explanation, we have examined the family of connectivity theories from a perspective that shifts from from labeling techniques as "connectivity analysis" and towards an approach that focuses on a construct (or set of constructs) around brain connectivity that have a substantive bridge to semantic information flows within the mind. In the process, we have examined multiple causal models, each attempting to explain certain features of the ASD phenotype. We have argued that, in the absence of an ability to cleanly permute connectivity in randomized fashion, the proposal and falsification of as many candidate causal models as possible for a particular behavioral explanandum within ASD is the best we can do toward establishing causality. In a few cases, we were able to identify specific areas where causal models can come into mutual exclusivity and to suggest a path forward.

This method of explaining one feature of ASD rather than trying to explain ASD as a whole sidesteps the problem of heterogeneity in this highly variable condition. However, future work will need to quilt together the surviving causal models that explain a plethora of behavioral phenomena in ASD. Maybe then we will be better able to understand the biology that enables such heterogeneity.

A complementary approach is to use behavioral, imaging and other inputs to develop datadriven clusters within ASD [129]. Such approaches are beginning to bear fruit in psychosis [130]. If the clusters are reproducible, we may then test causal models for the same behavioral explanandum, independently in each cluster (biotype). These clusters may eventually substitute for current DSM diagnoses [26].

A related issue is the poor discriminant validity of connectivity: several DSM diagnosis have been associated with the presence of altered connectivity [131]. The theory-driven study of altered connectivity in schizophrenia, for example, dates back well before even the original description of autism [31]. What does it mean that so many neuropsychiatric conditions have shown alterations of FC? Does it make it more likely that measured FC differences are due to a common source of artifact? Or may the FC differences be secondary to a host of neuropsychiatric bran pathologies? Or can multiple neuropsychiatric phenotypes-ASD, ADHD, schizophrenia and affective disorders - all truly be due to a single mechanism? Molecular research is confronted with the same challenge [132]. As in the case of binding together causal accounts for different behavioral explananda within the diagnosis of ASD, we will be tasked 
with piecing together causal accounts for similar (or different) explananda across DSM diagnoses.

An additional type of theoretical synthesis is across neurobiological accounts of ASD. Not all neurobiological theories are mutually exclusive; some may be compatible or even mutually supportive. Perhaps most relevant to long-range connectivity theories of ASD [42, 115] is the altered excitatory-inhibitory balance and GABAergic accounts of ASD [95]. As in the example of Roberts' and colleagues' work (see 1.3.2), multi-modality studies parsing both theories simultaneously may lead to the disproof of one theory, the subsumption of one theory under another, or the establishment of a more nuanced relationship between the two candidate mechanisms. We recall that the ongoing discussion around connectivity stated in the context of the excitatory-inhibitory imbalance hypothesis [54].

\subsubsection{Summary}

The goal of this chapter has been to engage neuroengineers into the cognitive neuroscientific discussion about how best to use their techniques to make true and substantiated inferences about the causal relationship between brain activity and behavior, both typical and clinical. To date, engineers have excelled at the development of novel techniques to measure brain physiology. And while it is the case that novel methods have greatly advanced theoretical developments in science [36], some neuroscientists have rung the alarm that excess enthusiasm over the latest imaging techniques has papered over the lack of necessary, careful consideration about how to interpret the data generated by these techniques in a nuanced and sophisticated way that will drive the field forward [2].

Cognitive neuroscience faces barriers other fields do not when it comes to causal inference. In most instances, we use the randomized, controlled trial. Yet in cognitive neuroscience, we have no way to randomly assign participants to a "high frontal-parietal connectivity" group vs. a "low frontal-parietal connectivity" group and assess whether or not a group develops ASD. Even if we had the technological means, such an approach is ethically inconceivable. So if we want to help individuals affected by neurobehavioral conditions by understanding the relevant biology, we need alternative approaches to causal inference. Statistical methods to modeling imaging and 
behavioral data may help $[78,133,134]$, but the approach we stress here is the generation of a densely populated domain of causal theories that can explain observed brain-behavior correlations, and the subsequent empiric testing of those theories, with the hopes of leaving only one causal graph standing.

To do so, we have recommended increasing the specificity of the elements of these causal graphs. We have proceeded by making explicit a variety of conceptualizations of connectivity that seem to be latent in the connectivity literature. We have proposed methods that may report on these sub-constructs of connectivity, but we further note that they still need to be rigorously validated [135]. Rather than basing a notion of connectivity on available methods, we have started with the construct and attempted to find methods that might report on those constructs.

Behavioral specificity results from ongoing work in carefully characterizing the behavioral phenotype of ASD, from clearly stating the behavioral explananda which the physiological investigation intends to explain, and from proposing which behavioral features should show statistical relationships with physiological data, and which should not. Computational models may help refine our theoretical notions of semantic information flow [63] and my further help link physiology and behavior.

While sharpening the specificity of observable metrics - connectivity analyses and behavioral measures - is a necessary step forward, these observable metrics need to be linked together via unobservable, theoretical constructs. Such constructs necessarily inherit the assumptions of the discipline from which they are derived. Here we are in a state of flux, both in regard to the connectivity literature and to the ASD literature. We have seen theories of ASD based on localizationist assumptions as well as those based on holist assumptions; not only is there no dominant "brain theory" associated with connectivity, but the advent of imaging connectivity measures has only served to recapitulate long-standing and never-resolved neuroscientific debates, such as those involving localizationism vs. holism. Explicitly testing localization assumptions against holist assumptions in different contexts, with our new methods, rather than merely treating them as ineffable axioms, will be necessary for progress in generating a general theory of brain function necessary to propel the field forward. The same is true for other sets of assumptions about brain function. Perhaps the best example we can provide of durable progress 
in this regard is that Rimland's speculation that cerebral connectivity is controlled by the function of the reticular formation has not stood the test of time.

It is also the case that the domain of ASD-specific connectivity theories is sparsely populated and therefore not inherently amenable to "either/or" hypothesis testing. We have presented theories that differ by localizationist/holist assumptions but also by varying developmental assumptions and by varying behavioral explananda. Nevertheless, even with this poor theoretical landscape, we have identified testable points of contact among some of the accounts, e.g., JUT's (2004) proposal that cognitive functions associated with any topography of macroscopic connectivity (as measured by fMRI) are specifically affected in ASD vs. JUT's (2012) proposal that cognitive function associated with frontal-parietal connectivity (as measured by fMRI) are specifically affected in ASD.

Engineering problems and scientific problems are inherently different things. In the former case, the performance of a tool often speaks for itself in a way that is observable to all. In the latter case, there is almost always the need for rigorous inference to interpret data as supporting or falsifying a hypothesis. We hope that this case study further invites neuroengineers into a conversation about the development and validation of cognitive neuroscientific techniques that is driven explicitly by theoretical needs. Such an approach is no more or less than the scientific method. 


\section{ACKNOWLEDGEMENTS}

We would like to thank Anastasios Bezerianos, PhD, Eric Chin, MD, Jim Pekar, PhD, Howard Egeth, $\mathrm{PhD}$, and Marcel Just, $\mathrm{PhD}$, for helpful comments on earlier drafts.

We would also like to thank Nitish Thakor, $\mathrm{PhD}$, and John Krakauer, MD, for their guidance on clarity of framing.

We especially thank Matthew Belmonte, $\mathrm{PhD}$, for his substantive thoughts on an earlier draft and for sharing a draft of an upcoming commentary regarding weak central coherence.

Any mistakes in reasoning are ours and not theirs. 


\section{References}

[1] T. Shallice, R. P. Cooper: The Organization of Mind, (Oxford University Press, Oxford, UK 2011)

[2] J. W. Krakauer, A. A. Ghazanfar, A. Gomez-Marin, M. A. MacIver, D. Poeppel: Neuroscience Needs Behavior: Correcting a Reductionist Bias, Neuron 93, 480-490 (2017)

[3] J. Morton: Understanding Developmental Disabilities: A Causal Model Approach, (Wiley-Blackwell, Singapore 2005)

[4] D. Marr: Vision: A Computational Investigation into the Human Representation and Processing of Visual Information, (MIT Press, Cambridge, MA 2010)

[5] M. R. Bennett, P. M. S. Hacker: Philosophical Foundations of Neuroscience, (Blackwell Publishing, Malden, MA 2003)

[6] E. Nagel: The Structure of Science: Problens in the Logic of Scientific Explanation, (Hackett Publishing Company, Inc, Indianapolis 1979)

[7] R. D. Mill, T. Ito, M. W. Cole: From Connectome to Cognition: The Search for Mechanism in Human Functional Brain Networks, Neuroimage 160, 124-139 (2017)

[8] D. t. A. : The Mystical Theology (Chapter 1). http://www.esoteric.msu.edu/VolumeII/MysticalTheology.html (c. 5-6 C BCE) Accessed February 1, 2020,

[9] R. A. Müller, P. Shih, B. Keehn, J. R. Deyoe, K. M. Leyden, D. K. Shukla: Underconnected, but How? A Survey of Functional Connectivity Mri Studies in Autism Spectrum Disorders, Cereb Cortex 21, 2233-2243 (2011)

[10] C. O'Reilly, J. D. Lewis, M. Elsabbagh: Is Functional Brain Connectivity Atypical in Autism? A Systematic Review of Eeg and Meg Studies, PLoS One 12, e0175870 (2017)

[11] G. Picci, S. J. Gotts, K. S. Scherf: A Theoretical Rut: Revisiting and Critically Evaluating the Generalized under/over-Connectivity Hypothesis of Autism, Dev Sci 19, 524-549 (2016) 
[12] P. Rane, D. Cochran, S. M. Hodge, C. Haselgrove, D. N. Kennedy, J. A. Frazier: Connectivity in Autism: A Review of Mri Connectivity Studies, Harv Rev Psychiatry 23, 223-244 (2015)

[13] H. Andreas: Theoretical Terms in Science. https:/plato.stanford.edu/archives/fall2017/entries/theoretical-terms-science/ (2017) Accessed January 112020

[14] D. Dennett: From Bacteria to Bach and Back: The Evolution of Minds, (W. W. Norton and Company, New York, NY 2017)

[15] L. Kanner: Autistic Disturbances of Affective Conduct, Nervous Child 2, 217-250 (1943)

[16] J. Baio: Prevalence of Autism Spectrum Disorders - Autism and Developmental Disabilities Monitoring Network, 14 Sites, United States, 2008, Morbidity and Mortality Weekly Report 61, 1-19 (2008)

[17] American Psychiatric Association: Diagnostic and Statistical Manual of Mental Disorders, 5th Edition: Dsm-5, (American Psychiatric Publishing, Arlington, VA 2013)

[18] E. L. Hill: Executive Dysfunction in Autism, Trends Cogn Sci 8, 26-32 (2004)

[19] S. H. Mostofsky, J. B. Ewen: Altered Connectivity and Action Model Formation in Autism Is Autism, Neuroscientist 17, 437-448 (2011)

[20] R. G. Winther: The Structure of Scientific Theories. https://plato.stanford.edu/archives/win2016/entries/structure-scientific-theories/ (2016) Accessed January 112020

[21] T. Shallice: From Neuropsychology to Mental Structure, (Cambridge University Press, Cambridge 1988)

[22] A. Lenartowicz, D. J. Kalar, E. Congdon, R. A. Poldrack: Towards an Ontology of Cognitive Control, Top Cogn Sci 2, 678-692 (2010)

[23] E. Hill: Evaluating the Theory of Executive Dysfunction in Autism, Developmental Review 24, 189-233 (2004) 
[24] F. Happé: Social and Nonsocial Development in Autism: Where Are the Links? In: The Development of Autism: Persepctives from Theory and Research, (Routledge, 2001), pp. 237-254

[25] S. Leekam: Social Cognitive Impairment and Autism: What Are We Trying to Explain?, Philos Trans R Soc Lond B Biol Sci 371, 20150082 (2016)

[26] L. Waterhouse, E. London, C. Gillberg: Asd Validity, Rev J Autism Dev Disord 3, (2016)

[27] T. R. Insel, B. N. Cuthbert: Endophenotypes: Bridging Genomic Complexity and Disorder Heterogeneity, Biol Psychiatry 66, 988-989 (2009)

[28] N. Geschwind: Disconnexion Syndromes in Animals and Man. Ii, Brain 88, 585-644 (1965)

[29] N. Geschwind: Disconnexion Syndromes in Animals and Man. I, Brain 88, 237-294 (1965)

[30] G. Goldenberg: Apraxia: The Cognitive Side of Motor Control, (Oxford University Pres, Oxford, UK 2013)

[31] G. Collin, E. Turk, M. P. van den Heuvel: Connectomics in Schizophrenia: From Early Pioneers to Recent Brain Network Findings, Biol Psychiatry Cogn Neurosci Neuroimaging 1, 199-208 (2016)

[32] J. B. Ewen, B. M. Lakshmanan, A. S. Pillai, D. McAuliffe, C. Nettles, M. Hallett, N. E. Crone, S. H. Mostofsky: Decreased Modulation of Eeg Oscillations in High-Functioning Autism During a Motor Control Task Frontiers in Human Neuroscience, doi:10.3389/fnhum.2016.00198 (2016)

[33] M. P. van den Heuvel, H. E. Hulshoff Pol: Exploring the Brain Network: A Review on Resting-State Fmri Functional Connectivity, Eur Neuropsychopharmacol 20, 519-534 (2010)

[34] O. Sporns, R. F. Betzel: Modular Brain Networks, Annu Rev Psychol 67, 613-640 (2016) 
[35] N. J. Kopell, H. J. Gritton, M. A. Whittington, M. A. Kramer: Beyond the Connectome: The Dynome, Neuron 83, 1319-1328 (2014)

[36] A. G. Greenwald: There Is Nothing So Theoretical as a Good Method, Perspect Psychol Sci 7, 99-108 (2012)

[37] L. Q. Uddin, K. Supekar, V. Menon: Reconceptualizing Functional Brain Connectivity in Autism from a Developmental Perspective, Front Hum Neurosci 7, 458 (2013)

[38] J. D. Power, K. A. Barnes, A. Z. Snyder, B. L. Schlaggar, S. E. Petersen: Spurious but Systematic Correlations in Functional Connectivity Mri Networks Arise from Subject Motion, Neuroimage 59, 2142-2154 (2012)

[39] R. A. Vasa, S. H. Mostofsky, J. B. Ewen: The Disrupted Connectivity Hypothesis of Autism Spectrum Disorders: Time for the Next Phase in Research, Biological Psychiatry: Cognitive Neuroscience and Neuroimaging 1, 245-252 (2016)

[40] Zhuangzi: Discussion on Making All Things Equal. In: The Complete Works of Zhuangzi, (c. 3rd cent. B.C.E./2013),

[41] B. Rimland: Infantile Autism: The Syndrome and Its Implications for a Neural Theory of Behaior, (Meredith Publishing Company, New York 1964)

[42] B. Horwitz, J. M. Rumsey, C. L. Grady, S. I. Rapoport: The Cerebral Metabolic Landscape in Autism. Intercorrelations of Regional Glucose Utilization, Arch Neurol 45, 749-755 (1988)

[43] C. M. Clark, R. Kessler, M. S. Buchsbaum, R. A. Margolin, H. H. Holcomb: Correlational Methods for Determining Regional Coupling of Cerebral Glucose Metabolism: A Pilot Study, Biol Psychiatry 19, 663-678 (1984)

[44] B. Horwitz, R. Duara, S. I. Rapoport: Intercorrelations of Glucose Metabolic Rates between Brain Regions: Application to Healthy Males in a State of Reduced Sensory Input, J Cereb Blood Flow Metab 4, 484-499 (1984)

[45] A. R. Damasio, R. G. Maurer: A Neurological Model for Childhood Autism, Arch Neurol 35, 777-786 (1978) 
[46] R. E. Jung, R. J. Haier: The Parieto-Frontal Integration Theory (P-Fit) of Intelligence: Converging Neuroimaging Evidence, Behav Brain Sci 30, 135-154; discussion 154-187 (2007)

[47] E. Courchesne, C. M. Karns, H. R. Davis, R. Ziccardi, R. A. Carper, Z. D. Tigue, H. J. Chisum, P. Moses, K. Pierce, C. Lord, A. J. Lincoln, S. Pizzo, L. Schreibman, R. H. Haas, N. A. Akshoomoff, R. Y. Courchesne: Unusual Brain Growth Patterns in Early Life in Patients with Autistic Disorder: An Mri Study, Neurology 57, 245-254 (2001)

[48] M. R. Herbert, D. A. Ziegler, C. K. Deutsch, L. M. O'Brien, N. Lange, A. Bakardjiev, J. Hodgson, K. T. Adrien, S. Steele, N. Makris, D. Kennedy, G. J. Harris, V. S. Caviness, Jr.: Dissociations of Cerebral Cortex, Subcortical and Cerebral White Matter Volumes in Autistic Boys, Brain 126, 1182-1192 (2003)

[49] M. R. Herbert, D. A. Ziegler, N. Makris, P. A. Filipek, T. L. Kemper, J. J. Normandin, H. A. Sanders, D. N. Kennedy, V. S. Caviness, Jr.: Localization of White Matter Volume Increase in Autism and Developmental Language Disorder, Ann Neurol 55, 530-540 (2004)

[50] K. Plunkett, A. Karmiloff-Smith, E. Bates, J. L. Elman, M. H. Johnson: Connectionism and Developmental Psychology, J Child Psychol Psychiatry 38, 53-80 (1997)

[51] M. A. Just, V. L. Cherkassky, T. A. Keller, N. J. Minshew: Cortical Activation and Synchronization During Sentence Comprehension in High-Functioning Autism: Evidence of Underconnectivity, Brain 127, 1811-1821 (2004)

[52] M. M. Mesulam: Large-Scale Neurocognitive Networks and Distributed Processing for Attention, Language, and Memory, Ann Neurol 28, 597-613 (1990)

[53] A. Luria: Higher Cortical Functions in Man, (Basic Books, Inc., New York 1966)

[54] M. K. Belmonte, E. H. Cook, Jr., G. M. Anderson, J. L. Rubenstein, W. T. Greenough, A. Beckel-Mitchener, E. Courchesne, L. M. Boulanger, S. B. Powell, P. R. Levitt, E. K. Perry, Y. H. Jiang, T. M. DeLorey, E. Tierney: Autism as a Disorder of Neural Information Processing: Directions for Research and Targets for Therapy, Mol Psychiatry 9, 646-663 (2004) 
[55] M. F. Casanova, D. P. Buxhoeveden, A. E. Switala, E. Roy: Minicolumnar Pathology in Autism, Neurology 58, 428-432 (2002)

[56] J. L. Rubenstein, M. M. Merzenich: Model of Autism: Increased Ratio of Excitation/Inhibition in Key Neural Systems, Genes Brain Behav 2, 255-267 (2003)

[57] U. Frith: Autism: Explaining the Enigma, (Basil Blackwell, 1989)

[58] L. Mottron, M. Dawson, I. Soulieres, B. Hubert, J. Burack: Enhanced Perceptual Functioning in Autism: An Update, and Eight Principles of Autistic Perception, J Autism Dev Disord 36, 27-43 (2006)

[59] K. Markram, H. Markram: The Intense World Theory - a Unifying Theory of the Neurobiology of Autism, Front Hum Neurosci 4, 224 (2010)

[60] J. S. Butler, S. Molholm, G. N. Andrade, J. J. Foxe: An Examination of the Neural Unreliability Thesis of Autism, Cerebral cortex 27, 185-200 (2017)

[61] L. A. Ajram, A. C. Pereira, A. M. S. Durieux, H. E. Velthius, M. M. Petrinovic, G. M. McAlonan: The Contribution of [1h] Magnetic Resonance Spectroscopy to the Study of Excitation-Inhibition in Autism, Prog Neuropsychopharmacol Biol Psychiatry 89, 236244 (2019)

[62] M. K. Belmonte, G. Allen, A. Beckel-Mitchener, L. M. Boulanger, R. A. Carper, S. J. Webb: Autism and Abnormal Development of Brain Connectivity, J Neurosci 24, 92289231 (2004)

[63] M. A. Just, T. A. Keller, V. L. Malave, R. K. Kana, S. Varma: Autism as a Neural Systems Disorder: A Theory of Frontal-Posterior Underconnectivity, Neurosci Biobehav Rev 36, 1292-1313 (2012)

[64] M. A. Just, V. L. Cherkassky, T. A. Keller, R. K. Kana, N. J. Minshew: Functional and Anatomical Cortical Underconnectivity in Autism: Evidence from an Fmri Study of an Executive Function Task and Corpus Callosum Morphometry, Cereb Cortex 17, 951-961 (2007) 
[65] F. Happé, R. Booth, R. Charlton, C. Hughes: Executive Function Deficits in Autism Spectrum Disorders and Attention-Deficit/Hyperactivity Disorder: Examining Profiles across Domains and Ages, Brain Cogn 61, 25-39 (2006)

[66] C. Hughes, J. Russell, T. W. Robbins: Evidence for Executive Dysfunction in Autism, Neuropsychologia 32, 477-492 (1994)

[67] H. Geurts, M. de Vries, S. van den Bergh: Executive Functioning Theory and Autism. In: Handbook of Executive Functioning, ed. by S. Goldstein, J. Naglieri (Springer, New York 2014),

[68] E. Courchesne: Brain Development in Autism: Early Overgrowth Followed by Premature Arrest of Growth, Ment Retard Dev Disabil Res Rev 10, 106-111 (2004)

[69] M. A. Just, S. Varma: The Organization of Thinking: What Functional Brain Imaging Reveals About the Neuroarchitecture of Complex Cognition, Cogn Affect Behav Neurosci 7, 153-191 (2007)

[70] B. Gepner, F. Feron: Autism: A World Changing Too Fast for a Mis-Wired Brain?, Neurosci Biobehav Rev 33, 1227-1242 (2009)

[71] J. Brock, C. C. Brown, J. Boucher, G. Rippon: The Temporal Binding Deficit Hypothesis of Autism, Dev Psychopathol 14, 209-224 (2002)

[72] P. L. Nunez: Toward a Quantitative Description of Large-Scale Neocortical Dynamic Function and Eeg, Behav Brain Sci 23, 371-398; discussion 399-437 (2000)

[73] P. J. Uhlhaas, W. Singer: Neural Synchrony in Brain Disorders: Relevance for Cognitive Dysfunctions and Pathophysiology, Neuron 52, 155-168 (2006)

[74] P. J. Uhlhaas, W. Singer: Abnormal Neural Oscillations and Synchrony in Schizophrenia, Nat Rev Neurosci 11, 100-113 (2010)

[75] P. J. Uhlhaas, G. Pipa, B. Lima, L. Melloni, S. Neuenschwander, D. Nikolic, W. Singer: Neural Synchrony in Cortical Networks: History, Concept and Current Status, Front Integr Neurosci 3, 17 (2009)

[76] J. Pearl: Causality, second ed., (Cambridge University Press, Cambridge 2009) 
[77] R. A. Poldrack: Inferring Mental States from Neuroimaging Data: From Reverse Inference to Large-Scale Decoding, Neuron 72, 692-697 (2011)

[78] D. McAuliffe, K. Hirabayashi, J. H. Adamek, Y. Luo, D. Crocetti, A. S. Pillai, Y. Zhao, N. E. Crone, S. H. Mostofsky, J. B. Ewen: Increased Mirror Overflow Movements in Adhd Are Associated with Altered Eeg Alpha/Beta Band Desynchronization, Eur J Neurosci, doi:10.1111/ejn.14642 (2019)

[79] C. E. Shannon: Communication in the Presence of Noise, Proceedings of the Institute of Radio Engineers 37, 10-21 (1949)

[80] D. Hartline, D. Colman: Rapid Conduction and the Evolution of Giant Axons and Myelinated Fibers, Current Biology 17, R29-R35 (2007)

[81] M. A. Cohen, D. C. Dennett, N. Kanwisher: What Is the Bandwidth of Perceptual Experience?, Trends in cognitive sciences 20, 324-335 (2016)

[82] E. K. Miller, T. J. Buschman: Working Memory Capacity: Limits on the Bandwidth of Cognition, Daedalus 144, 112-122 (2015)

[83] A. Mazzoni, N. K. Logothetis, S. Panzer: The Information Content of Local Field Potentials: Experiments and Models. In: Principles of Neural Coding, ed. by R. Q. Quiroga, S. Panzeri (CRC Press, Boca Raton, FL 2013),

[84] A. N. Nielsen, C. Gratton, J. A. Church, N. U. F. Dosenbach, K. J. Black, S. E. Petersen, B. L. Schlaggar, D. J. Greene: Atypical Functional Connectivity in Tourette Syndrome Differs between Children and Adults, Biol Psychiatry, doi:10.1016/j.biopsych.2019.06.021 (2019)

[85] S. H. Baum, R. A. Stevenson, M. T. Wallace: Testing Sensory and Multisensory Function in Children with Autism Spectrum Disorder, J Vis Exp, doi:10.3791/52677e52677 (2015)

[86] C. C. Haswell, J. Izawa, L. R. Dowell, S. H. Mostofsky, R. Shadmehr: Representation of Internal Models of Action in the Autistic Brain, Nat Neurosci 12, 970-972 (2009) 
[87] J. Izawa, S. E. Pekny, M. K. Marko, C. C. Haswell, R. Shadmehr, S. H. Mostofsky: Motor Learning Relies on Integrated Sensory Inputs in Adhd, but over-Selectively on Proprioception in Autism Spectrum Conditions, Autism Res 5, 124-136 (2012)

[88] T. A. Knaus, A. M. Silver, M. Kennedy, K. A. Lindgren, K. C. Dominick, J. Siegel, H. Tager-Flusberg: Language Laterality in Autism Spectrum Disorder and Typical Controls: A Functional, Volumetric, and Diffusion Tensor Mri Study, Brain Lang 112, 113-120 (2010)

[89] S. K. Sundaram, A. Kumar, M. I. Makki, M. E. Behen, H. T. Chugani, D. C. Chugani: Diffusion Tensor Imaging of Frontal Lobe in Autism Spectrum Disorder, Cereb Cortex 18, 2659-2665 (2008)

[90] T. L. Johnson, A. Pillai, D. McAuliffe, S. Mostofsky, J. Ewen: Children with Autism Spectrum Disorders Showtiming-Related Changes During Praxis Execution, Ann Neurol 78, S163 (2015)

[91] L. A. Wheaton, G. Nolte, S. Bohlhalter, E. Fridman, M. Hallett: Synchronization of Parietal and Premotor Areas During Preparation and Execution of Praxis Hand Movements, Clin Neurophysiol 116, 1382-1390 (2005)

[92] N. M. Gage, B. Siegel, T. P. Roberts: Cortical Auditory System Maturational Abnormalities in Children with Autism Disorder: An Meg Investigation, Brain Res Dev Brain Res 144, 201-209 (2003)

[93] T. P. Roberts, S. Y. Khan, M. Rey, J. F. Monroe, K. Cannon, L. Blaskey, S. Woldoff, S. Qasmieh, M. Gandal, G. L. Schmidt: Meg Detection of Delayed Auditory Evoked Responses in Autism Spectrum Disorders: Towards an Imaging Biomarker for Autism, Autism Research 3, 8-18 (2010)

[94] T. P. L. Roberts, J. Matsuzaki, L. Blaskey, L. Bloy, J. C. Edgar, M. Kim, M. Ku, E. S. Kuschner, D. Embick: Delayed M50/M100 Evoked Response Component Latency in Minimally Verbal/Nonverbal Children Who Have Autism Spectrum Disorder, Mol Autism 10, 34 (2019) 
[95] J. Rubenstein, M. M. Merzenich: Model of Autism: Increased Ratio of Excitation/Inhibition in Key Neural Systems, Genes, Brain and Behavior 2, 255-267 (2003)

[96] J. I. Berman, L. Bloy, L. Blaskey, E. S. Kuschner, M. Ku, M. Dipiero, L. D. Gaetz, J. C. Edgar, T. P. Roberts: Multimodal Stratification of Asd with the Help of Dti, Meg, and Mrs, Proc. International Society for Autism Research, (2019)

[97] A. S. Ghuman, J. R. McDaniel, A. Martin: A Wavelet-Based Method for Measuring the Oscillatory Dynamics of Resting-State Functional Connectivity in Meg, Neuroimage 56, 69-77 (2011)

[98] M. Hassan, F. Wendling: Electroencephalography Source Connectivity: Aiming for High Resolution of Brain Networks in Time and Space, IEEE Signal Processing Magazine 35, $81-96$ (2018)

[99] B. He, L. Astolfi, P. A. Valdes-Sosa, D. Marinazzo, S. Palva, C. G. Benar, C. M. Michel, T. Koenig: Electrophysiological Brain Connectivity: Theory and Implementation, IEEE Transactions on Biomedical Engineering, (2019)

[100] K. Mahjoory, V. V. Nikulin, L. Botrel, K. Linkenkaer-Hansen, M. M. Fato, S. Haufe: Consistency of Eeg Source Localization and Connectivity Estimates, Neuroimage 152, 590-601 (2017)

[101] G. Van Hoey, R. Van de Walle, B. Vanrumste, M. D'Havse, I. Lemahieu, P. Boon: Beamforming Techniques Applied in Eeg Source Analysis, Proc. ProRISC99 10, 545549 (1999)

[102] A. S. Hincapie, J. Kujala, J. Mattout, A. Pascarella, S. Daligault, C. Delpuech, D. Mery, D. Cosmelli, K. Jerbi: The Impact of Meg Source Reconstruction Method on SourceSpace Connectivity Estimation: A Comparison between Minimum-Norm Solution and Beamforming, Neuroimage 156, 29-42 (2017)

[103] R. B. Stein, E. R. Gossen, K. E. Jones: Neuronal Variability: Noise or Part of the Signal?, Nature Reviews Neuroscience 6, 389 (2005)

[104] K. Wiesenfeld, F. Moss: Stochastic Resonance and the Benefits of Noise: From Ice Ages to Crayfish and Squids, Nature 373, 33 (1995) 
[105] F. Jaramillo, K. Wiesenfeld: Mechanoelectrical Transduction Assisted by Brownian Motion: A Role for Noise in the Auditory System, Nature neuroscience 1, 384 (1998)

[106] I. Dinstein, D. J. Heeger, L. Lorenzi, N. J. Minshew, R. Malach, M. Behrmann: Unreliable Evoked Responses in Autism, Neuron 75, 981-991 (2012)

[107] S. Dakin, U. Frith: Vagaries of Visual Perception in Autism, Neuron 48, 497-507 (2005)

[108] D. R. Simmons, A. E. Robertson, L. S. McKay, E. Toal, P. McAleer, F. E. Pollick: Vision in Autism Spectrum Disorders, Vision research 49, 2705-2739 (2009)

[109] G. Winterer, D. R. Weinberger: Genes, Dopamine and Cortical Signal-to-Noise Ratio in Schizophrenia, Trends Neurosci 27, 683-690 (2004)

[110] L. Hu, A. Mouraux, Y. Hu, G. D. Iannetti: A Novel Approach for Enhancing the Signalto-Noise Ratio and Detecting Automatically Event-Related Potentials (Erps) in Single Trials, Neuroimage 50, 99-111 (2010)

[111] A. Arazi, Y. Yeshurun, I. Dinstein: Neural Variability Is Quenched by Attention, Journal of Neuroscience 39, 5975-5985 (2019)

[112] S. J. Webb, R. Bernier, H. A. Henderson, M. H. Johnson, E. J. Jones, M. D. Lerner, J. C. McPartland, C. A. Nelson, D. C. Rojas, J. Townsend, M. Westerfield: Guidelines and Best Practices for Electrophysiological Data Collection, Analysis and Reporting in Autism, J Autism Dev Disord, doi:10.1007/s10803-013-1916-6 (2013)

[113] J. B. Ewen, J. A. Sweeney, W. Z. Potter: Conceptual, Regulatory and Strategic Imperatives in the Early Days of Eeg-Based Biomarker Validation for Neurodevelopmental Disabilities, Front. Integr. Neurosci., doi:10.3389/fnint.2019.00045 (2019)

[114] P. J. Uhlhaas, F. Roux, E. Rodriguez, A. Rotarska-Jagiela, W. Singer: Neural Synchrony and the Development of Cortical Networks, Trends Cogn Sci 14, 72-80 (2010)

[115] P. J. Uhlhaas, W. Singer: What Do Disturbances in Neural Synchrony Tell Us About Autism?, Biol Psychiatry 62, 190-191 (2007) 
[116] D. C. Rojas, K. Maharajh, P. Teale, S. J. Rogers: Reduced Neural Synchronization of Gamma-Band Meg Oscillations in First-Degree Relatives of Children with Autism, BMC Psychiatry 8, 66 (2008)

[117] T. W. Wilson, D. C. Rojas, M. L. Reite, P. D. Teale, S. J. Rogers: Children and Adolescents with Autism Exhibit Reduced Meg Steady-State Gamma Responses, Biol Psychiatry 62, 192-197 (2007)

[118] H. Fujisaka, T. Yamada: Stability Theory of Synchronized Motion in Coupled-Oscillator Systems, Progress of theoretical physics 69, $32-47$ (1983)

[119] L. M. Pecora, T. L. Carroll: Synchronization in Chaotic Systems, Physical review letters 64, 821 (1990)

[120] G. Pfurtscheller, C. Andrew: Event-Related Changes of Band Power and Coherence: Methodology and Interpretation, J Clin Neurophysiol 16, 512-519 (1999)

[121] C. Stam, B. Van Dijk: Synchronization Likelihood: An Unbiased Measure of Generalized Synchronization in Multivariate Data Sets, Physica D: Nonlinear Phenomena 163, 236251 (2002)

[122] Y. A. Pijnenburg, Y. v d Made, A. M. van Cappellen van Walsum, D. L. Knol, P. Scheltens, C. J. Stam: Eeg Synchronization Likelihood in Mild Cognitive Impairment and Alzheimer's Disease During a Working Memory Task, Clin Neurophysiol 115, 13321339 (2004)

[123] J. P. Lachaux, E. Rodriguez, J. Martinerie, F. J. Varela: Measuring Phase Synchrony in Brain Signals, Hum Brain Mapp 8, 194-208 (1999)

[124] N. F. Rulkov, M. M. Sushchik, L. S. Tsimring, H. D. Abarbanel: Generalized Synchronization of Chaos in Directionally Coupled Chaotic Systems, Physical Review E 51, 980 (1995)

[125] T. Schreiber: Interdisciplinary Application of Nonlinear Time Series Methods, Physics reports 308, 1-64 (1999) 
[126] A. S. Pillai, D. McAuliffe, B. M. Lakshmanan, S. H. Mostofsky, N. E. Crone, J. B. Ewen: Altered Task-Related Modulation of Long-Range Connectivity in Children with Autism, Autism Res, doi:10.1002/aur.1858 (2017)

[127] R. W. Emerson, C. Adams, T. Nishino, H. C. Hazlett, J. J. Wolff, L. Zwaigenbaum, J. N. Constantino, M. D. Shen, M. R. Swanson, J. T. Elison, S. Kandala, A. M. Estes, K. N. Botteron, L. Collins, S. R. Dager, A. C. Evans, G. Gerig, H. Gu, R. C. McKinstry, S. Paterson, R. T. Schultz, M. Styner, I. Network, B. L. Schlaggar, J. R. Pruett, Jr., J. Piven: Functional Neuroimaging of High-Risk 6-Month-Old Infants Predicts a Diagnosis of Autism at 24 Months of Age, Sci Transl Med 9, (2017)

[128] Š. Holiga, J. F. Hipp, C. H. Chatham, P. Garces, W. Spooren, X. L. D'Ardhuy, A. Bertolino, C. Bouquet, J. K. Buitelaar, C. Bours, A. Rausch, M. Oldehinkel, M. Bouvard, A. Amestoy, M. Caralp, S. Gueguen, M. Ly-Le Moal, J. Houenou, C. F. Beckmann, E. Loth, D. Murphy, T. Charman, J. Tillmann, C. Laidi, R. Delorme, A. Beggiato, A. Gaman, I. Scheid, M. Leboyer, M. A. d'Albis, J. Sevigny, C. Czech, F. Bolognani, G. D. Honey, J. Dukart: Patients with Autism Spectrum Disorders Display Reproducible Functional Connectivity Alterations, Sci Transl Med 11, (2019)

[129] M. V. Lombardo, M. C. Lai, S. Baron-Cohen: Big Data Approaches to Decomposing Heterogeneity across the Autism Spectrum, Mol Psychiatry 24, 1435-1450 (2019)

[130] B. A. Clementz, J. A. Sweeney, J. P. Hamm, E. I. Ivleva, L. E. Ethridge, G. D. Pearlson, M. S. Keshavan, C. A. Tamminga: Identification of Distinct Psychosis Biotypes Using Brain-Based Biomarkers, Am J Psychiatry 173, 373-384 (2016)

[131] D. M. Barch, C. S. Carter: Functional and Structural Brain Connectivity in Psychopathology, Biol Psychiatry Cogn Neurosci Neuroimaging 1, 196-198 (2016)

[132] M. J. Gandal, J. R. Haney, N. N. Parikshak, V. Leppa, G. Ramaswami, C. Hartl, A. J. Schork, V. Appadurai, A. Buil, T. M. Werge, C. Liu, K. P. White, C. CommonMind, E. C. Psych, P.-B. W. G. i, S. Horvath, D. H. Geschwind: Shared Molecular Neuropathology across Major Psychiatric Disorders Parallels Polygenic Overlap, Science 359, 693-697 (2018) 
[133] A. D. Passaro, J. M. Vettel, J. McDaniel, V. Lawhern, P. J. Franaszczuk, S. M. Gordon: A Novel Method Linking Neural Connectivity to Behavioral Fluctuations: BehaviorRegressed Connectivity, J Neurosci Methods 279, 60-71 (2017)

[134] Q. Yu, B. B. Risk, K. Zhang, J. S. Marron: Jive Integration of Imaging and Behavioral Data, Neuroimage 152, 38-49 (2017)

[135] M. E. Strauss, G. T. Smith: Construct Validity: Advances in Theory and Methodology, Annu Rev Clin Psychol 5, 1-25 (2009) 
Table 1: Summary of Long-Range Causal Accounts in Section 1.2

\begin{tabular}{|c|c|c|c|c|}
\hline & Explanandum & $\begin{array}{c}\text { FC } \\
\text { Sub-Construct }\end{array}$ & Localization & Role of Development \\
\hline $\begin{array}{l}\text { Horwitz et } \\
\text { al. [39] }\end{array}$ & $\begin{array}{l}\text { Internally- vs. } \\
\text { externally- } \\
\text { directed } \\
\text { attention }\end{array}$ & & Frontal-Parietal & \\
\hline $\begin{array}{l}\text { Courchesne } \\
\text { et al. [44] }\end{array}$ & & & Agnostic & $\begin{array}{l}\text { White matter develops } \\
\text { prematurely, without proper } \\
\text { influence of the environment }\end{array}$ \\
\hline $\begin{array}{l}\text { Herbert et } \\
\text { al. }[45-46]\end{array}$ & & & $\begin{array}{l}\text { Holist (anti- } \\
\text { localizationist) }\end{array}$ & \\
\hline $\begin{array}{l}\text { JUT (2004) } \\
{[48]}\end{array}$ & $\begin{array}{l}\text { Low- vs. high- } \\
\text { level cognition }\end{array}$ & & $\begin{array}{l}\text { Focal vs. } \\
\text { distributed }\end{array}$ & \\
\hline $\begin{array}{l}\text { JUT (2012) } \\
{[59]}\end{array}$ & Planning & Bandwidth & Frontal-Parietal & $\begin{array}{l}\text { Decreased influence of frontal } \\
\text { areas on parietal areas causes } \\
\text { secondarily increased } \\
\text { autonomy of parietal regions }\end{array}$ \\
\hline TSPD [66] & Multiple & $\begin{array}{l}\text { Time-sensitive } \\
\text { synchronization }\end{array}$ & Agnostic & \\
\hline
\end{tabular}

$F C=$ Functional connectivity; JUT = Just's et al. Underconnectivity Theory; TSPD = TemporoSpatial Processing Disorder 